\title{
Riding the Bubble with Convex Incentives*
}

\author{
Juan Sotes-Paladino ${ }^{\dagger}$ \\ Fernando Zapatero ${ }^{\ddagger}$ \\ Department of Finance \\ Marshall School of Business \\ The University of Melbourne University of Southern California
}

May 2018

\begin{abstract}
We show that benchmark-linked convex incentives can lead risk-averse money managers aware of mispricing to over-invest in overpriced securities. In the model, the managers' risk-seeking behavior varies in response to the interaction of mispricing with convexity and benchmarking concerns. Convexity effects can exacerbate the manager's over-investment in overvalued non-benchmark securities. In contrast, they potentially offset benchmarking effects studied in the literature, leading to under-investment in overpriced benchmark securities. Under correlated mispricing across assets, our model rationalizes positive positions in non-benchmark, negative-risk premium (i.e., "bubble") securities and "pairs trading" in two overvalued securities. Our findings help explain several empirical puzzles.
\end{abstract}

Keywords: Money management, convex incentives, benchmarking, mispricing.

JEL Classification: D81, D82, D83, G11, G23.

\footnotetext{
*We thank the comments of participants at the 2014 Jerusalem Finance Conference, the 2014 EFA Meetings, the 2014 ANU's RSFAS Summer Camp, the 2016 AFA Meetings, the Second International Congress on Actuarial Science and Quantitative Finance and the 2017 Finance Forum, especially the respective discussants, Alon Raviv, Igor Makarov, Norman Schurhöff, Andrea Buffa and Maurizio Montone. We also thank Bruce Grundy and seminar participants at the University of Melbourne and the Central Bank of Chile for their comments. Finally, we are grateful to two anonymous referees and Stijn Van Nieuwerburgh (the Editor) for many constructive comments that greatly helped to improve the quality of the paper. Existing errors are our responsibility.

${ }^{\dagger}$ E-mail:juan.sotes@unimelb.edu.au.

${ }^{\ddagger}$ E-mail: fzapatero@marshall.usc.edu.
} 


\section{Introduction}

The role of institutional investors in enhancing financial markets efficiency has come under increased scrutiny following recent episodes of severe-perceived-mispricing. Several empirical studies document that mutual funds, and especially hedge funds, were heavily invested in technology stocks during the "tech bubble" of the late 1990s and early 2000s. ${ }^{1}$ The evidence seems particularly puzzling in light of the high power of the convex incentives commonly adopted in the asset management industry, which reward good performance but do not penalize (or do so to a lesser extent) bad outcomes. Following a standard argument, such incentives should encourage managers to trade more aggressively against mispricing. ${ }^{2}$ The argument should be more compelling in the case of sophisticated investors such as hedge funds, which follow absolute-return strategies and thus have no benchmarking concerns with respect to mispriced assets. ${ }^{3}$

In this paper, we tackle this puzzle within a dynamic trading model that considers convexity and benchmarking features of the money managers' incentives. In the model, sophisticated riskaverse managers observe assets' mispricing. Under standard incentives, they would generally trade against it. However, under convex incentives their optimal trading can be substantially different, as the managers' risk-seeking behavior varies in response to the interaction of mispricing with convexity and benchmarking concerns. Based on this interaction, we derive several novel results. First, we are able to rationalize the empirically documented over-investment of managers in overvalued assets that do not belong to their benchmark. In fact, we show that convexity effects can more than offset the portfolio tilt toward benchmark assets found by previous studies. Thus, our second result is that convex incentives can lead to aggressive trading against overvalued asset that are included in the benchmark. Third, we show that in response to convex incentives and their current relative performance managers might increase their allocation in an asset as its overvaluation worsens. Fourth, we establish that a manager can optimally hold

\footnotetext{
1 See, e.g., Brunnermeier and Nagel (2004), Greenwood and Nagel (2009), and Griffin, Harris, Shu, and Topaloglu (2011).

${ }^{2}$ For an exposition of this argument and an empirical investigation of its validity, see Dass, Massa, and Patgiri (2008).

${ }^{3}$ Buffa, Vayanos, and Woolley (2014) rationalize asset managers' excess demand for overpriced securities as induced by endogenous benchmarking concerns.
} 
a "bubble"-i.e., negative-risk premium—asset. ${ }^{4}$ Fifth, accounting for correlated mispricing, we rationalize empirically observed "pairs trading" in two overvalued assets, a strategy that finances the purchase of a relatively less overvalued asset by selling short the more overvalued. ${ }^{5}$ Lastly, we show that in equilibrium the trading of the managers can exacerbate overvaluation relative to the case of no convex incentives.

Convex incentives are ubiquitous in the money management industry. Among hedge funds, convexity arises from their typical fee structure. This structure includes a flat management fee plus a "bonus" performance fee - typically, several times larger than the management feeover profits in excess of a hurdle performance rate or a high-water mark. In the mutual fund industry, implicit convexities in manager's incentives can result from the asymmetric relation between a fund's performance relative to a benchmark, usually a market index, and its clients' share purchases and redemptions. A further source of convexity in the incentives of mutual fund managers is the prevalence of bonus payments in the end-of-year compensation packages. ${ }^{6}$

We explore the consequences of this type of incentives in a continuous-time frictionless securities market over a finite investment horizon. The general version of our model features multiple risky assets and a risk-less asset. Risky assets are subject to mispricing, in the sense that their observed prices can differ from fundamental value. Mispricing is temporary, as it converges to zero by the end of the investment period. The resulting asset returns can be characterized as the sum of a "fair" - i.e., fundamental - and an "abnormal" - the asset's "alpha" - expected returns. Whereas the fair return reflects compensation for exposure to systematic risk, alpha is directly related to the extent of mispricing. We follow prior literature in characterizing alphas as possibly stochastic and mean reverting. If multiple assets are mispriced, alphas can be correlated across assets. In this framework, we study the trading problem of a sophisticated

\footnotetext{
${ }^{4}$ We follow the definition of "bubble" assets in DeMarzo, Kaniel, and Kremer (2008).

${ }^{5}$ For an examination of pairs trading strategies see, e.g., Gatev, Goetzmann, and Rouwenhorst (2006).

${ }^{6}$ The convexity in mutual funds' implicit incentives has been documented by, e.g., Chevalier and Ellison (1997) and Sirri and Tufano (1998). The convexity in their compensation packages is documented by Farnsworth and Taylor (2006) and Ma, Tang, and Gomez (2015). Other forces in the mutual fund industry seem to attenuate this convexity, though. Ibert, Kaniel, Nieuwerburgh, and Vestman (2017) find that the relation between manager's compensation and their funds' assets under management is concave instead, and the sensitivity of their pay to performance is weak.
} 
investor (a money manager) who observes both fundamentals and prices - hence, is aware of the mispricing - and has convex incentives. The convexity follows from a "bonus" fee payment contingent on outperforming a given benchmark (fixed-weight) portfolio of the assets.

Our first contribution is to characterize in closed form the optimal portfolio of the manager under multi-asset risky mispricing. This is a technically challenging problem, as it entails finding the solution to a dynamic optimization problem under both kinked incentives and a stochastic - as implied by the risky feature of mispricing - investment opportunity set. To the best of our knowledge, ours is the first analytical characterization of the optimal portfolio in such an environment. ${ }^{7}$

The optimal portfolio consists of the "desired," or normal portfolio that a manager with no convex or benchmark-linked incentives would select, plus three additional components. ${ }^{8}$ The sum of these components determines the manager's over- or under-investment in each asset relative to the case of standard incentives. The first component is a hedging demand against the added absolute performance risk to which the manager is exposed by the sensitivity of incentives to fund performance, and tilts the portfolio toward a more conservative position in the risky assets than the normal policy. The second is a hedging demand against the relative performance risk to which the manager is exposed when the compensation is benchmark adjusted, and consists of a positive allocation to the benchmark portfolio. The third is a "risk-shifting" demand, specific to convex incentives, that captures the manager's risk-seeking ("gambling") behavior to finish ahead of the benchmark. This component assigns a large positive weight to assets that are underrepresented in the benchmark relative to the normal portfolio, and a large negative weight to assets that are overrepresented. ${ }^{9}$ Crucially, whether an asset in our setting is under- or overrepresented in the benchmark depends not only on the benchmark's (given) composition or the manager's risk aversion, but also on the level of mispricing. In particular, an asset's weight in the normal portfolio falls as its overpricing rises. Thus, for an overvalued non-benchmark asset,

\footnotetext{
7 For the type of kinked incentives in our framework, Basak and Makarov (2014) regard optimal dynamic policies under a stochastic investment opportunity set as analytically intractable (see, e.g., their footnote 5).

${ }^{8}$ From now on, we call "desired" the composition of the normal portfolio.

${ }^{9}$ See Basak, Pavlova, and Shapiro (2007).
} 
the normal allocation can initially exceed the benchmark allocation (i.e., because the asset is excluded from the benchmark), but fall below right after a large enough overvaluation shock. The initially underrepresented asset becomes overrepresented in the benchmark after the shock, and the manager's gamble switches from a long to a short position in the asset.

Our second contribution is to show that convex incentives can lead a manager to adopt opposite trading stances in an overvalued asset depending on whether it belongs or not to the benchmark. This result can be illustrated in a single-risky asset (a "stock") economy. If the stock does not belong to the benchmark, the manager can optimally overweight it for low to intermediate levels of overvaluation. The reason is that, as long as the overvaluation is not too severe, the zero weight of the stock in the benchmark is too low relative to the manager's normal allocation. To outperform the benchmark, the manager bets on future scenarios of rising overvaluation by aggressively increasing the allocation to the stock. For the same low-to-intermediate overvaluation levels, an all-stock benchmark assigns, relative to the manager's normal portfolio, an excessively high weight to the stock. To outperform the benchmark, the manager bets on future scenarios of declining overvaluation by aggressively reducing the allocation to the stock. In this case, convexity effects can more than offset the tilt of the relative-performance hedging demand toward an overvalued benchmark stock.

As a third contribution, we find that the interaction between incentive convexity and the manager's up-to-date relative performance gives rise to new trading patterns. For instance, a manager can increase the allocation to a non-benchmark stock as its overpricing worsens, even when the normal portfolio reduces it. Intuitively, a surge in overvaluation that improves the relative performance of an underperforming manager can exacerbate her risk-seeking behavior. When the stock is underrepresented in the benchmark relative to the normal portfolio, the manager takes an even greater gamble on a future scenario of rising stock overvaluation. As a result, she can end up overweighting the overvalued non-benchmark stock more than a standard investor unaware of the overpricing.

These results can rationalize a number of "puzzles" regarding the trading strategy of sophisticated investors without recurring to behavioral arguments. The opposing effects of convex 
incentives on the manager's portfolio depending on the benchmark can reconcile the seemingly contradicting findings of recent empirical studies. For instance, Brunnermeier and Nagel (2004) find that managers with convex incentives but no stock benchmark, such as hedge fund managers, were heavily exposed to overpriced stocks during the tech bubble. By contrast, Massa and Patgiri (2009) find that similar incentive convexity induced mutual fund managers, typically benchmarked against a stock index, to trade aggressively against overvalued stocks during the same period. The demand for an overvalued non-benchmark stock in response to the interaction of convexity and up-to-date performance can explain the allocation of overpriced tech stocks in hedge funds' portfolios in excess of the market portfolio, as documented by Brunnermeier and Nagel (2004). ${ }^{10}$

Our fourth contribution is to examine the effects of convex incentives under multi-asset mispricing. With multiple risky assets, some or all of them can be mispriced or belong to the benchmark, and mispricing can be stochastic and correlated across assets. Strikingly, we find that under correlated mispricing a manager can optimally allocate a positive weight to a non-benchmark, bubble asset, that the normal investor sells short. This result does not hold in a single-asset market or in a multi-asset market with the constant investment opportunity set of prior literature, where the risk-shifting demand always sells negative risk-premium, nonbenchmark assets, which the normal policy sells short. Under correlated mispricing, when the manager can trade in two overvalued assets the effect arises from the combination of cross-asset diversification (substitution) and convexity effects. As the relative overvaluation of one of the assets (i.e., its overvaluation in excess of the other asset's overvaluation) worsens, an investor can reallocate money between the two assets (i.e., substitute the relatively less overvalued asset for the more overvalued one) to exploit the relative mispricing while limiting portfolio exposure to mispricing risk. Risk aversion can prevent a normal investor from taking advantage of the relative mispricing aggressively, and the substitution effect just translates into changes in the relative sizes of two short positions. However, the managers' risk-shifting demand in response to

\footnotetext{
${ }^{10}$ Our results are also consistent with evidence from the experimental work in Holmen, Kirchler, and Kleinlercher (2014), who find that trading at inflated prices is rational for subjects with convex incentives.
} 
convex incentives can exacerbate this substitution effect to the point of inducing a long position in the relatively less overvalued, possibly non-benchmark asset. The resulting strategy resembles the long-short "pairs trading" popular among hedge funds.

Our findings under multi-asset mispricing help explain additional puzzles. First, Liu and Timmermann (2013) characterize pairs trading strategies as suboptimal over short horizons for a risk-averse investor with standard incentives. Our results rationalize the adoption of these strategies in practice by risk-averse investor with convex incentives (e.g., hedge funds). Second, our findings help explain the empirically documented net positive positions in bubble assets of some institutional investors (Griffin, Harris, Shu, and Topaloglu (2011)) during the tech bubble. Third, they help explain the over-investment in technology stocks during the same episode by mutual fund managers benchmarked against non-tech stocks, as documented by Greenwood and Nagel (2009).

Our fifth contribution is to examine the price impact of convex incentives in an equilibrium setting. We show that managers with convex incentives can exacerbate overpricing even beyond the levels prevailing in an economy entirely populated by uninformed investors that overestimate fundamentals. More generally, we show that convex incentives exacerbate mispricing (both overand undervaluation) relative to the case in which convex incentives are absent.

From a methodological perspective, our paper is closest to the literature on money managers' risk taking in response to non-standard incentives. We build on Basak, Pavlova, and Shapiro (2007) and extend their analysis to a setup in which risky assets are subject to stochastic and potentially correlated mispricing. Our equilibrium model is based on the setting of Cuoco and Kaniel (2011). The manager's investment in securities with negative risk premium in our model extends results in Makarov and Plantin (2014) to a setting with risk-averse agents. In general equilibrium models without convex incentives, Vayanos and Woolley (2013) and Buffa, Vayanos, and Woolley (2014) find that money managers can push prices of benchmark stocks away from fundamental value. Malamud and Petrov (2014) study the effects of convex incentives on price informativeness and volatility under risk-neutrality.

Our paper also contributes to the theoretical literature on limits to arbitrage (Allen and 
Gorton (1993), Shleifer and Vishny (1997), Abreu and Brunnermeier (2003), Liu and Longstaff (2004), Stein (2009), Sato (2009)). This literature relates a manager's over-investment in overvalued securities to issues of asymmetric information, share redemption or synchronization risks, capital constraints or crowded trades. Our findings complement these explanations using only the type of compensation arrangements observed in practice.

The paper is structured as follows. In Section 2 we derive the optimal trading of money managers with convex incentives under mispricing in a single-risky asset market. We characterize the managers' optimal trading in multiple mispriced assets in Section 3. In Section 4 we examine the price impact of these strategies in general equilibrium. We close the paper with some conclusions in Section 5. We present all proofs in Appendix A, and discuss further aspects of our model in Appendices B, C, as well as in an Internet Appendix.

\section{Convex Incentives and Trading in a Mispriced Asset}

\subsection{Financial Markets}

We consider a continuous-time frictionless securities market over a finite investment horizon $[0, T]$. The general specification of our model features $n+2$ securities $(n \geq 0)$, consisting of one risk-less asset and $n+1$ risky assets. Asset prices and fundamentals, as defined below, are exogenously given. The risk-less asset pays a constant interest rate $r$ per unit of time and is fairly priced. The risky assets can be mispriced, meaning that their observed prices can differ from fundamental value. For ease of exposition, we present the case of a single risky security market in this section, and defer the general case of multiple risky securities to Section 3.

In the single risky security market, the risky asset (henceforth, the "stock") has observed price $S$ and fundamental value $F$. Let $r_{t}^{S} \equiv d \log \left(S_{t}\right)$ and $r_{t}^{F} \equiv d \log \left(F_{t}\right)$ denote, respectively, the growth in log stock price and fundamental value. We assume that the stock's mean return $E_{t}\left[r_{t}^{S}\right] / d t$ consists of the "fair" (or "normal") expected return $\tilde{r}_{t} \equiv E_{t}\left[r_{t}^{F}\right] / d t$, and a possibly non-zero "abnormal" expected return $\alpha_{t}$ (the stock's "alpha"). Letting the Brownian motion $B$ 
be the source of risk in the economy, the (log) stock price and fundamental value follow:

$$
\begin{aligned}
& r_{t}^{S}=\left(\tilde{r}_{t}+\alpha_{t}\right) d t+\sigma_{t} d B_{t}, \\
& r_{t}^{F}=\tilde{r}_{t} d t+\delta d B_{t},
\end{aligned}
$$

for return volatility $\sigma_{t}>0$, as specified below, and constant fundamental volatility $\delta>0$. The fair mean rate of return in excess of the risk-free rate, $\tilde{r}_{t}+1 / 2 \sigma_{t}^{2}-r$, is the compensation for the stock's exposure to (systematic) risk, $\gamma \sigma_{t}^{2}$, where $\gamma>0$ reflects the average relative risk aversion in the underlying economy. We denote $\tilde{\mu}_{t} \equiv r+\gamma \sigma_{t}^{2}$ and $\mu_{t} \equiv \tilde{\mu}_{t}+\alpha_{t}$, respectively, the resulting fair and observed mean rates of returns on the stock.

In specifying the stock's abnormal expected return, we build on the empirical findings of, e.g., Poterba and Summers (1988) and Brennan and Wang (2010), to posit an autoregressive process of order one, $\operatorname{AR}(1)$, for $\alpha_{t}:{ }^{11}$

$$
\begin{aligned}
& d \alpha_{t}=-2 v_{t} \alpha_{t} d t-\sigma_{t} v_{t} d B_{t} \\
& d v_{t}=-v_{t}^{2} d t
\end{aligned}
$$

for given initial values $\alpha_{0}$ and $v_{0} \geq 0$. The specified dynamics (3) and (4) for $\alpha_{t}$, along with our specification of $\sigma_{t}$ below, allow for explicit solutions while ensuring realistic statistical properties (e.g., alpha mean-reverting to zero for suitable choices of $\left.v_{0}\right)$.

We assume that the volatility of abnormal expected returns magnifies the stock return volatility relative to fundamental volatility through:

$$
\sigma_{t}=\delta\left(1+v_{t} \tau\right)
$$

where $\tau \equiv T-t$ is the time remaining to the end of the period. Following Dumas, Kurshev, and

\footnotetext{
11 The autoregressive structure of mispricing and, more generally, returns is also predicted by theoretical models such as those of Madhavan and Smidt (1993), Wang (1993) and Johnson (2004). Note that, to ensure market completeness, the same source of risk $B$ drives the uncertainty in both (1) and (3), implying perfect correlation between $r^{S}$ and $d \alpha$. We relax this assumption in the multi-asset setup of Section 3.
} 
Uppal (2009), specification (5) can be interpreted as capturing "excessive" asset price volatility. In our model, excess volatility is induced by the component $v_{t}$ of the volatility in abnormal expected returns. ${ }^{12}$ Because this component affects abnormal expected returns but not fundamental value, we say that $v_{t}$ reflects mispricing risk.

Under these assumptions, we show in Appendix A the existence of a process $F$ such that the levels of stock price and fundamental value are related via:

$$
\begin{aligned}
S_{t} & =F_{t} \exp \left(-\delta \frac{\alpha_{t}}{\sigma_{t}} \tau\right), \\
& =F_{t} \exp \left(O V_{t}\right) .
\end{aligned}
$$

According to Eq. (6), $O V_{t} \equiv-\delta \alpha_{t} \tau / \sigma_{t}$ reflects the extent of stock mispricing, with $O V_{t}>0$ corresponding to stock overvaluation and $O V_{t}<0$ to undervaluation. The mispricing is temporary, as prices converge to fundamental value no later than $t=T(\tau=0) .{ }^{13}$ The extent of over- or undervaluation $O V_{t}$ is proportional to the stock's "appraisal ratio" $\alpha_{t} / \sigma_{t}$. Holding $\delta$, $\sigma_{t}$ and $\tau$ constant, stock overpricing $O V_{t}>0$ (underpricing $O V_{t}<0$ ) necessarily translates, as argued by Johnson (2004), into a negative (positive) abnormal expected return $\alpha_{t}$. Conversely, a negative (respectively, positive) abnormal expected return $\alpha_{t}$ implies stock overvaluation (undervaluation), $O V>0(O V<0)$, whereas a zero $\alpha_{t}$ means that the stock is fairly priced $(O V=0)$.

We note that whether mispricing in the model is stochastic (risky) or deterministic (riskless) depends on $v_{0}$. If $v_{0}=0$, the stock's alpha is constant at $\alpha_{t}=\alpha_{0}$ for all $t$ in $[0, T]$, and mispricing $O V_{t}$ converges deterministically to zero at $T$. If, on the other hand, $v_{0}>0$, the stock's alpha mean-reverts to zero following a stochastic path to convergence. We assume that mispricing is risky $\left(v_{0}>0\right)$ throughout, relegating the analysis of risk-less mispricing to Internet Appendix A.

\footnotetext{
12 In this sense, our analysis can also be thought of as characterizing the optimal portfolio under convex incentives and excess volatility. We thank a referee for this observation.

13 The main implications of the model remain unaltered when we assume instead that final convergence takes place at some later $T^{*}>T$, such that $S_{T^{*}}=F_{T^{*}}$.
} 


\subsection{The Money Manager's Problem}

The money manager receives an end-of-period compensation $W_{T}^{M}$, which is the product of a fee rate $f$ and the value at $T$ of the fund's assets under management (AUM) $W_{T} \cdot{ }^{14}$ The manager dynamically chooses the time- $t$ fraction $\phi_{t}$ of $W_{t}$ that is allocated to the stock. Given $W_{0}=w_{0}$, the value of the portfolio (AUM) follows:

$$
d W_{t}=\left(r+\phi_{t}\left(\mu_{t}-r\right)\right) W_{t} d t+\phi_{t} \sigma_{t} W_{t} d B_{t}
$$

The compensation fee $f_{T}$ is a function of the manager's performance relative to a benchmark index $Y$ (henceforth just "benchmark"). For initial value $Y_{0}=y_{0}$, the benchmark is the value of a portfolio investing a fixed fraction $\phi^{Y} \in[0,1]$ in the stock and the rest in the risk-free asset:

$$
d Y_{t}=\left(r+\phi^{Y}\left(\mu_{t}-r\right)\right) Y_{t} d t+\phi^{Y} \sigma_{t} Y_{t} d B_{t}
$$

The fee rate $f_{T}$ is specified as the following 2-piece function:

$$
f_{T}=k\left(\frac{R_{T}}{\bar{\zeta} R_{T}^{Y}}\right)^{\psi_{1}} \mathbb{1}_{\left\{R_{T}<\bar{\zeta} R_{T}^{Y}\right\}}+k\left(\frac{R_{T}}{\bar{\zeta} R_{T}^{Y}}\right)^{\psi_{2}} \mathbb{1}_{\left\{R_{T} \geq \bar{\zeta} R_{T}^{Y}\right\}}
$$

where $k, \bar{\zeta}>0,0 \leq \psi_{1} \leq \psi_{2}, R_{T} \equiv W_{T} / W_{0}$, and $R_{T}^{Y} \equiv Y_{T} / Y_{0}$. Specification (9) generalizes the flow-performance function in Basak and Makarov (2014), and is similar to the incentive function in Kaniel and Kondor (2013).

The fee rate (9) is positive and (weakly) increasing in the manager's relative performance $R_{T} / R_{T}^{Y}$. Let $\zeta \equiv \bar{\zeta} W_{0} / Y_{0}$ denote the (normalized) performance threshold, and $\mathcal{R}_{1} \equiv\left\{R_{T}<\bar{\zeta} R_{T}^{Y}\right\}=$ $\left\{W_{T}<\zeta Y_{T}\right\}$ and $\mathcal{R}_{2} \equiv\left\{R_{T} \geq \bar{\zeta} R_{T}^{Y}\right\}=\left\{W_{T} \geq \zeta Y_{T}\right\}$ denote, respectively, the end-of-period underperformance and outperformance regions. If the performance sensitivity $\psi_{1}$ in $\mathcal{R}_{1}$ is smaller than the slope $\psi_{2}$ in $\mathcal{R}_{2}$, the relationship is asymmetric and presents a convex kink at $R_{T}=\bar{\zeta} R_{T}^{Y}$. In practice, managers face kinked incentives when their compensation includes bonus payments

\footnotetext{
${ }^{14}$ In the spirit of, e.g., Basak and Makarov (2014) we take the compensation contract as given. For an equilibrium model endogenizing the amount of AUM, or the contract between institutions and their investors, see Vayanos and Woolley (2013) and Buffa, Vayanos, and Woolley (2014).
} 
for relatively good performance (see references in the Introduction). The compensation function can be symmetric, yet convex at $R_{T}=\bar{\zeta} R_{T}^{Y}$, also if $1<\psi_{1}=\psi_{2}$. To fix the terminology, we refer only to kinked fee schedules as "convex." 15

The fee function (9) is general enough to capture the incentives of different financial intermediaries such as hedge funds (HF) and mutual funds (MF). Hedge funds typically follow so-called "absolute return" strategies, whereby incentive fees depend on performance against a money market rate such as 3-month LIBOR (i.e., $\phi^{Y}=0$ ) plus a spread. By contrast, mutual funds typically follow so-called "relative return" strategies, whereby their performance is benchmarked against a broad stock market index (e.g., $\phi^{Y}=1$ ). In Appendix B we discuss how (9) can be calibrated to the typical incentives of HF or MF managers. ${ }^{16}$ In Section 2.4 we compare the optimal responses of $\mathrm{HF}$ and MF managers to their respective incentives to rationalize existing evidence on their trading.

The manager has constant relative risk aversion (CRRA) preferences with relative risk aversion (RRA) coefficient $\gamma>1$, and maximizes expected utility from final wealth. ${ }^{17}$ Managers choose the fraction $\hat{\phi}_{t}$ of AUM $W_{t}$ allocated to the stock to maximize:

$$
E_{0}\left[\frac{\left(W_{T}^{M}\right)^{1-\gamma}}{1-\gamma}\right]
$$

subject to initial assets under management $w_{0}$ and the self-financing constraint (7).

\subsection{Optimal Investment Strategy}

Markets are complete in this economy (one risky asset $S$ driven by one Brownian motion $B$ ). Absence of arbitrage opportunities requires the existence of a unique market price of risk $\eta=$

\footnotetext{
${ }^{15}$ Likewise, $f_{T}$ could be concave at any other point $R_{T} \neq \bar{\zeta} R_{T}^{Y}$ (e.g., if $0 \leq \psi_{i}<1, i \in\{1,2\}$ ) yet we refer to it as convex as long as $\psi_{1}<\psi_{2}$. We thank a referee for this observation. Arguably, the case $0 \leq \psi_{1}<\psi_{2}$ is a better representation than the case $1<\psi_{1}=\psi_{2}$ of the type of bonus packages prevalent in the financial industry, which trigger bonus payments only if a certain performance goal is achieved. We nevertheless compare these two cases in Appendix C.

${ }^{16}$ For simplicity of exposition, we assume $\psi_{1}=0$ in most of our analysis. However, we allow for $\psi_{1}>0$ in our analysis of benchmarking versus incentive convexity of Appendix C.

17 The assumption implies that the manager's coefficient of relative risk aversion equals the "aggregate" risk aversion reflected in asset prices, as introduced in Section 2.1.
} 
$\sigma^{-1}(\mu-r)$. Since $\mu=\tilde{\mu}+\alpha, \eta$ consists of the sum of the market price of risk under no mispricing, $\tilde{\eta}_{t} \equiv \sigma_{t}^{-1}\left(\tilde{\mu}_{t}-r\right)$, plus the appraisal ratio $\kappa_{t} \equiv \sigma_{t}^{-1} \alpha_{t}$ :

$$
\eta_{t}=\tilde{\eta}_{t}+\kappa_{t}
$$

The manager then sees financial markets as driven by a unique state-price deflator (SPD)

$$
\pi_{t}=\exp \left\{-r t-\frac{1}{2} \int_{0}^{t} \eta_{s}^{2} d s-\int_{0}^{t} \eta_{s} d B_{s}\right\}
$$

\subsubsection{Benchmark Case: Investment Policy without Convex Incentives}

To assess the effects of convex incentives on the manager's trading strategy, we first examine the investment policy of a standard investor with no convex incentives $\left(\psi_{1}=\psi_{2}=0\right)$. Following Basak, Pavlova, and Shapiro (2007), we refer to this as the normal policy. We denote $\phi_{t}^{N}$ the time- $t$ portfolio weight in the stock for a normal investor. Sotes-Paladino (2017) characterizes $\phi_{t}^{N}$ as:

$$
\begin{aligned}
\phi_{t}^{N} & =\frac{1}{\frac{1+v_{t} \tau / \gamma}{1+v_{t} \tau}} \frac{\eta_{t}}{\gamma \sigma_{t}} \\
& =\frac{1}{\frac{1+v_{t} \tau / \gamma}{1+v_{t} \tau}} \frac{\tilde{\mu}_{t}-r+\alpha_{t}}{\gamma \sigma_{t}^{2}}
\end{aligned}
$$

The normal policy (12) is consistent with the commonly expected behavior of a sophisticated risk-averse trader who detects and trades against the asset mispricing. Relative to the case of no mispricing ( $\alpha_{t}=0$ in Eq. (12)), the normal portfolio underweights the stock when it is overvalued $\left(\alpha_{t}<0\right)$ and overweights it when it is undervalued $\left(\alpha_{t}>0\right)$. The overweighting or underweighting increase with the magnitude of the mispricing $\left|\alpha_{t}\right|$. For severe levels of overpricing, akin to a negative risk premium $\left(-\alpha_{t}<\tilde{\mu}_{t}-r\right)$, the normal policy sells the stock short $\left(\phi_{t}^{N}<0\right)$. In the next subsection we contrast this with the behavior of a sophisticated trader with convex incentives - the money manager. 


\subsubsection{The Investment Policy of the Money Manager}

Compared to standard incentives, performance-sensitive incentives increase the effective relative risk aversion from $\gamma$ - the intrinsic relative risk aversion- to $\gamma_{i} \equiv \gamma+\psi_{i}(\gamma-1)>\gamma$, depending on performance with respect to the benchmark. ${ }^{18}$ Under performance-sensitive and convex incentives $\left(\psi_{1}<\psi_{2}\right)$, effective relative risk aversion increases more in the outperformance region $\mathcal{R}_{2}$ than in the underperformance region $\mathcal{R}_{1}$. Based on this interaction between relative performance and risk aversion, we characterize the manager's optimal trading strategy in the following:

Proposition 1. For $t \in[0, T]$, the manager's time-t optimal holding of the stock $\hat{\phi}_{t}$ is:

$$
\hat{\phi}_{t}=\phi_{t}^{N}-\left[\sum_{i=1}^{2} \frac{\hat{W}_{i, t}}{\hat{W}_{t}} \frac{1-\gamma / \gamma_{i}}{1+v_{t} / \gamma_{i} \tau}\right] \phi_{t}^{N}+\left[\sum_{i=1}^{2} \frac{\hat{W}_{i, t}}{\hat{W}_{t}}\left(1-\frac{\gamma}{\gamma_{i}}\right)\right] \phi^{Y}+\sum_{i=1}^{2} \frac{\hat{W}_{i, t}}{\hat{W}_{t}} \frac{\Phi_{i}\left(t, \phi_{t}^{N}-\phi^{Y}\right)}{\Pi_{i, t}}
$$

and optimal assets under management are $\hat{W}_{t}=\hat{W}_{1, t}+\hat{W}_{2, t}$, where for $i=1,2, \hat{W}_{i, t} \equiv$ $\pi_{t}^{-1} E_{t}\left[\pi_{T} \hat{W}_{i, T}\right]$ is characterized in Appendix A,

$$
\begin{gathered}
\Phi_{i}\left(t, \phi_{t}^{N}-\phi^{Y}\right) \equiv \frac{(-1)^{i-1}}{\sigma_{t} \sqrt{\tau}} \sqrt{\frac{1+v_{t} \tau}{1+\frac{v_{t}}{\gamma_{i}}}}\left(n\left(\bar{d}_{i, t}\right)-n\left(\underline{d}_{i, t}\right)\right), \\
\underline{d}_{i, t} \equiv \gamma \sqrt{\frac{1+v_{t} \tau}{1+\frac{v_{t}}{\gamma_{i}} \tau}} \frac{1+\frac{v_{t}}{\gamma} \tau}{v_{t} \sqrt{\tau}} \delta\left(\phi_{\gamma, t}^{N}-\phi^{Y}\right)-\sqrt{\frac{1+\frac{v_{t}}{\gamma_{i} \tau}}{v_{t} \tau}} \Gamma, \quad \bar{d}_{i, t} \equiv \underline{d}_{i, t}+2 \sqrt{\frac{1+\frac{v_{t}}{\gamma_{i}}}{v_{t} \tau}} \Gamma,
\end{gathered}
$$

$\Pi_{1, t} \equiv N\left(\underline{d}_{1, t}\right)+1-N\left(\bar{d}_{1, t}\right), \Pi_{2, t} \equiv N\left(\bar{d}_{2, t}\right)-N\left(\underline{d}_{2, t}\right), N(\cdot)$ is the standard normal cumulative distribution function, $n(\cdot)$ is the standard normal density function, $\lambda_{M}>0$ solves $\hat{W}_{0}=w_{0}$, and $\Gamma \geq 0$ is given in Appendix A.

Proposition 1 shows that the optimal investment strategy of the manager has four components: the normal policy and three additional terms that capture deviations from it, denoted as $A P H_{t} \equiv-\sum_{i=1}^{2}\left[\hat{W}_{i, t} / \hat{W}_{t}\left(1-\gamma / \gamma_{i}\right) /\left(1+v_{t} / \gamma_{i} \tau\right)\right] \phi_{t}^{N}, R P H_{t} \equiv \sum_{i=1}^{2}\left[\hat{W}_{i, t} / \hat{W}_{t}\left(1-\gamma / \gamma_{i}\right)\right] \phi^{Y}$ and $R S D_{t} \equiv \sum_{i=1}^{2} \hat{W}_{i, t} / \hat{W}_{t} \Phi_{i}\left(t, \phi_{t}^{N}-\phi^{Y}\right) / \Pi_{i, t}$. We next describe each of these terms.

\footnotetext{
18 This effect is identified in Basak and Makarov (2014). Intuitively, for $\psi_{i}>0$, variations in before-fee AUM are magnified on an after-fee basis by the fee rate $k\left(W_{T} / \zeta Y_{T}\right)^{\psi_{i}}$. Therefore, the manager's compensation fluctuates more in response to the same change in before-fees AUM than under standard incentives, raising the manager's effective risk aversion over her intrinsic risk aversion.
} 
$A P H$ stands for absolute performance hedge. It responds to the increase in effective risk aversion induced by the sensitivity of the manager's incentives to the fund performance, and tilts the portfolio toward a more conservative position in the stock than the normal policy. ${ }^{19}$ Indeed, the manager's payoff $W_{T}^{M}=f_{T} W_{T}$ displays a higher sensitivity to absolute performance $R_{T}$ than the payoff of an identical normal investor. This implies that, compared to the normal investor, the manager is overexposed to absolute performance risk. To hedge this risk, she reduces the normal allocation to the stock via the "contrarian" $A P H$ demand. The magnitude of $A P H$ rises with the performance sensitivity of incentives $\psi_{1}, \psi_{2}$ and hence, when incentives are convex $\left(\psi_{1}<\psi_{2}\right)$, is larger (in absolute value) for outperforming managers. This demand is independent of the benchmark - therefore similar for different types of managers (e.g., MF and HF managers) - and varies with the level of mispricing in the opposite direction from the normal policy.

$R P H$ is the relative performance hedge. Accounting for the risk that the manager faces when deviating from the benchmark, $R P H$ is a positive allocation to the benchmark portfolio. ${ }^{20}$ Specifically, the manager's incentives $f_{T}$ induce negative sensitivity of compensation $W_{T}^{M}$ to the performance $R_{T}^{Y}$ of the benchmark, whereby an increase in $R_{T}^{Y}$ reduces $W_{T}^{M}$. The manager hedges this relative performance risk by increasing, compared to an otherwise identical investor with no benchmark concerns - i.e., the normal investor, the allocation to the benchmark. Like $A P H$, this "benchmarking effect" intensifies with the performance sensitivity $\psi_{1}, \psi_{2}$, implying, when incentives are convex, a larger $R P H$ demand for outperforming managers. Unlike $A P H$, this demand is benchmark specific - it varies across managers according to their benchmarksand is unaffected by the stock mispricing.

Finally, $R S D$ is the risk-shifting demand. It reflects the manager's risk-seeking ("gambling") behavior to finish ahead of the benchmark in response to convex and benchmark-linked incentives, as first studied by Basak, Pavlova, and Shapiro (2007). Intuitively, RSD can represent

\footnotetext{
19 By definition, $0 \leq \hat{W}_{i, t} \leq \hat{W}_{t}, 0 \leq \gamma \leq \gamma_{i}$, and $v_{t} \tau \geq 0$. Hence, the second term on the right-hand side of Eq. (13) has the opposite sign of $\phi_{t}^{N}$ and a smaller absolute value.

${ }^{20}$ From footnote 19, the coefficient multiplying $\phi^{Y}$ in the third term of the right-hand side of Eq. (13) is positive and less than one in absolute value.
} 
a large long position in the stock whenever it has a lower weight in the benchmark than in the manager's desired portfolio - the normal policy (i.e., the stock is underrepresented in the benchmark), and a large short position otherwise (the stock is overrepresented in the benchmark). In our model, in contrast to Basak, Pavlova, and Shapiro (2007), even for the same benchmark and managerial risk aversion, this demand can adopt both long and short positions in the stock at different mispricing levels. The reason is that the manager's desired allocation to the stock, but not the benchmark allocation, varies with the level of mispricing. ${ }^{21}$ In particular, it can exceed the benchmark allocation (e.g., if the stock is excluded from the benchmark) for a given initial stock overvaluation but fall below right after a large enough overvaluation shock. Accordingly, the manager's gamble will switch from a long to a short position in the stock. We argue below that, at certain overvaluation levels, this gambling motive can drive the manager's over-investment in the stock, or adopt opposite signs depending on whether the stock belongs or not to the benchmark.

\subsection{The Money Manager's Trading Under Mispricing}

Under stochastic mispricing, fluctuations in the stock over- or undervaluation over the investment period affect not only the available investment opportunities but also the manager's standing relative to the benchmark. As argued above, relative performance affects the manager's effective risk aversion and, as a result, also the $A P H, R P H$ and $R S D$ demands. In this subsection, we analyze the manager's investment by distinguishing between the effects of mispricing and relative performance on the optimal portfolio. To do so, we first examine the manager's trading as of the start of the period $(t=0)$, when relative performance is given. We then study how the dynamic interplay between the two channels determines the optimal trading at an interim period $(0<t<T)$.

In both cases, we focus our analyses on two popular benchmarks in the money management industry; namely, a risk-free portfolio $\left(\phi^{Y}=0\right)$ and an all-stock portfolio $\left(\phi^{Y}=1\right)$. Following

${ }^{21}$ This can be seen from Eq. (15), where the thresholds $\bar{d}_{i, t}$ and $\underline{d}_{i, t}$ that determine $R S D$ depend on the sign and magnitude of the difference $\phi_{t}^{N}-\phi^{Y}$, which in turn depends on the stock's abnormal expected return $\alpha_{t}$ through Eq. (12). 
our discussion in Section 2.2 (and Appendix B), we associate the risk-less benchmark $\phi^{Y}=0$ with hedge funds (HF) and the risky benchmark $\phi^{Y}=1$ with mutual funds (MF).

\subsubsection{Initial Holdings of a Mispriced Stock}

Figure 1 illustrates how, in the presence of mispricing, convex incentives lead money managers to deviate from the normal policy. In panel 1(a) we plot the initial weight of the stock in the money managers' and normal portfolios for different overvaluation scenarios. We decompose the managers' excess investment in the stock relative to the normal policy into its $A P H, R P H$ and $R S D$ components in panels $1(\mathrm{~b})$ and $1(\mathrm{c}) .{ }^{22}$ Panel $1(\mathrm{a})$ shows that the managers' policies across overvaluation scenarios are not uniform, as they do not unambiguously trade more aggressively against mispricing than the normal policy. On the contrary, their initial excess positions in the overvalued stock are non-linear functions of the severity of the mispricing.

The managers' trading against mispricing differs substantially depending on whether the stock belongs to the benchmark (MF) or not (HF). As previously explained, the $A P H$ demand does not vary between the HF and the MF managers of Panels 1(b) and 1(c). Thus, the different stances of the $\mathrm{HF}$ and MF managers against mispricing must arise from the relative performance or the risk-shifting considerations captured, respectively, by the $R P H$ and $R S D$ demands. Prior studies have shown that the first channel - relative performance concerns - can generate excessive investment in a benchmark (Basak and Pavlova (2013)), even if it is overvalued (Buffa, Vayanos, and Woolley (2014)). In Fig. 1, the RPH demand differs between Panel 1(b) and Panel 1(c) depending on the managers' benchmarks. In line with these studies the benchmarking demand $R P H$ is positive and large for the MF manager at all levels of mispricing, whereas it is zero for the HF manager.

More surprisingly, the convexity effects embedded in the risk-shifting demand $R S D$ can induce very different bets against mispricing depending on a manager's benchmark. Indeed, whereas the $\mathrm{HF}$ and MF managers of Panels 1(b) and 1(c) engage in risk shifting by taking

\footnotetext{
${ }^{22}$ In the Figure, the performance sensitivity parameters of both hedge and mutual funds are calibrated to the convexity of incentives in the hedge fund industry, as detailed in Appendix B.1. Market and risk aversion parameters are typical and in line with prior literature (e.g., Brennan and Xia (2001)).
} 


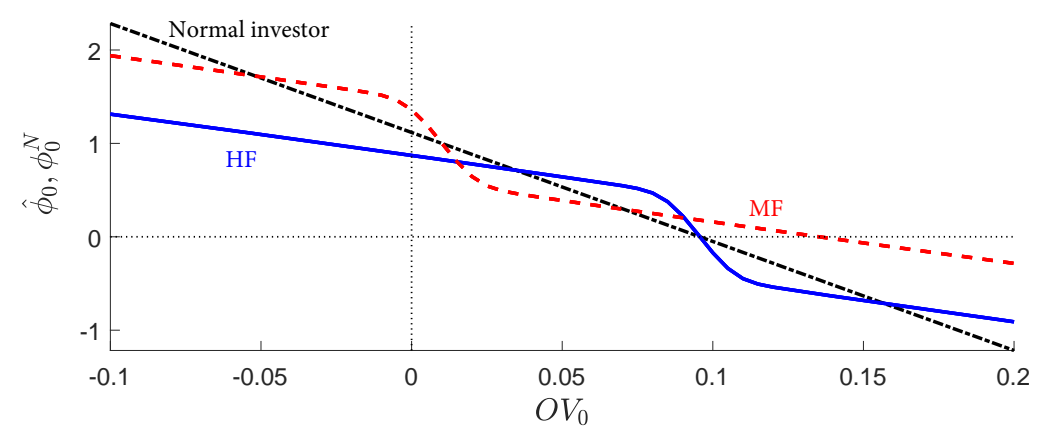

(a) Initial Portfolio

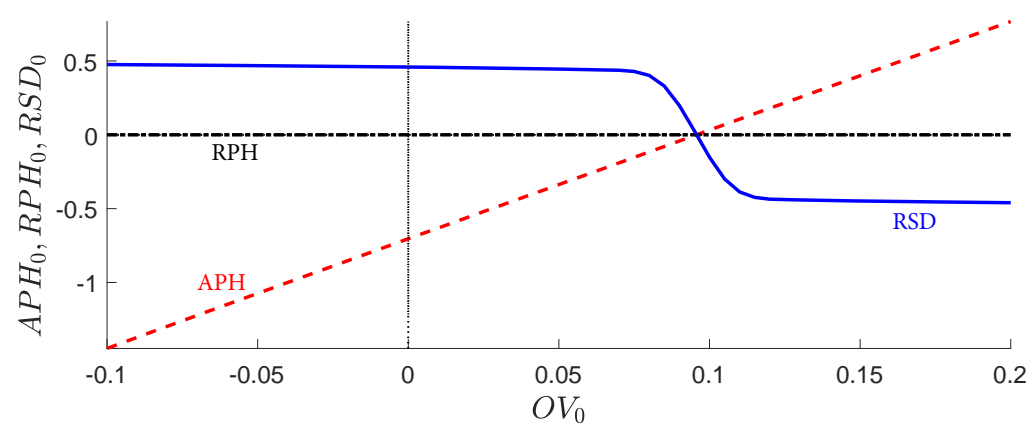

(b) $A P H, R P H$ and $R S D$ in a HF Manager's Initial Portfolio

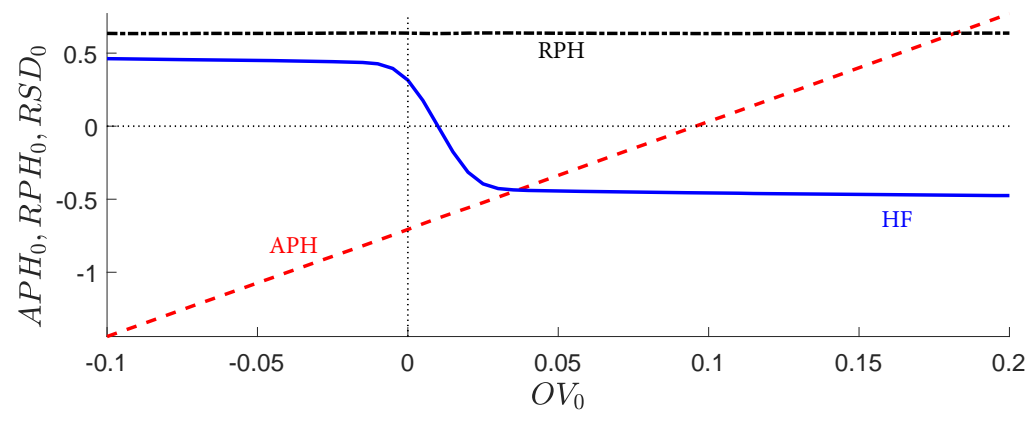

(c) $A P H, R P H$ and $R S D$ in a MF Manager's Initial Portfolio

Figure 1: Initial Portfolio Weight in a Mispriced Stock

In panel 1(a) the solid blue, dashed red and dash-and-dot black lines represent the initial portfolio weights in the stock for different levels of initial overvaluation $O V_{0}$ of, respectively, a hedge fund (HF) manager, a mutual fund (MF) manager, and a normal investor. In panels $1(\mathrm{~b})$ and $1(\mathrm{c})$ the solid blue, dashed red and dash-and-dot black lines represent, respectively, the $R S D, A P H$ and $R P H$ components of the optimal initial portfolios of the HF (panel 1(b)) and the MF (panel 1(c)) managers of panel 1(a). Parameter values are: $\psi_{1}=0, \psi_{2}=5, \bar{\zeta}=1, \gamma=5, T=1, r=1.5 \%, \delta=.129, v_{0}=0.15$.

aggressive bets against both undervaluation and severe overvaluation, their $R S D$ demands have opposite signs at intermediate overvaluation levels. ${ }^{23}$ In particular, $R S D$ is positive for the HF

23 Throughout, we describe the severity of mispricing (e.g., "high overvaluation") by comparison with the assumed mispricing parameters. In this section, we assume an instantaneous annualized volatility of changes in overvaluation $O V_{0}$ of approximately $2 \%$. Thus, an overvaluation level of $9 \%$ can be considered "high" as long as it is several standard deviations above the expected initial mispricing. This would be the case, for example, if the expected initial overvaluation in the economy were $0 \%$, in which case an observed value of $9 \%$ would be 4.5 standard deviations above the expectation. 
manager but negative for the MF manager. As observed in Fig. 1(a), the effect can lead the HF manager to overinvest in the overvalued, non-benchmark stock. Equally strikingly, it can also, along with a negative $A P H$ demand, more than offset benchmarking effects and lead the MF manager to underinvest in the same overvalued stock when it belongs to the benchmark.

This result can be understood as follows. As argued above, the direction of the manager's gamble depends on the allocation of the stock in the manager's desired-normal — portfolio relative to its allocation in the benchmark portfolio. Following the intuition in Basak, Pavlova, and Shapiro (2007), whenever the normal portfolio weight in the stock exceeds the benchmark's $\left(\phi_{t}^{N}>\phi^{Y}\right.$, i.e., the stock is underrepresented in the benchmark), the manager gambles on outperforming in future scenarios of rising overvaluation by aggressively increasing the allocation to the stock. In our model, unlike in Basak, Pavlova, and Shapiro (2007), a rising overvaluation will not only improve the manager's relative standing but will also reduce the stock weight in the normal portfolio, eventually pushing it below the benchmark weight $\left(\phi_{t}^{N}<\phi^{Y}\right)$. When this occurs, the manager switches to gambling on outperforming in future scenarios of declining overvaluation by aggressively decreasing the weight in the stock instead. Clearly, the level of overpricing necessary to trigger this change decreases with the stock weight in the benchmark portfolio. In particular, for the same intermediate overvaluation levels, the stock can be underrepresented in a no-stock benchmark $\left(\phi^{Y}=0\right)$ and simultaneously overrepresented in an all-stock benchmark $\left(\phi^{Y}=1\right)$. The respective managers' risk-shifting demands will take opposite signs and lead to the contrasting trading stances of Fig. 1(a).

Thus, accounting for the interaction of convexity and benchmarking effects in the incentives of managers leads to two surprising patterns in their holdings of a mispriced stock. First, a manager with no stock-related performance concerns might optimally overweight a mispriced stock, relative to the normal policy, for a wide range of overvaluation. Second, and conversely, similar convex incentives can lead a manager with performance concerns relative to an overvalued stock (the MF manager) to aggressively underweight it for the same overvaluation levels.

These results can reconcile the apparently conflicting findings of recent empirical studies. On the one hand, Brunnermeier and Nagel (2004) report that many HF managers were heavily 
exposed to overpriced stocks during the so-called "tech bubble" of the late 1990s. On the other hand, Massa and Patgiri (2009) hypothesize and empirically verify that, among U.S. mutual fund managers, higher convexity in incentives led to more aggressive trading against stock overvaluation during the same episode. Since in practice HF managers typically face incentives with higher convexity than MF managers, ${ }^{24}$ the former finding seems inconsistent with the finding of Massa and Patgiri (2009). The conflicting evidence is consistent, however, with the opposing effects of convex incentives on the trading of the HF and MF managers in our model. Ignoring the convexity in incentives, benchmarking considerations alone are unlikely to explain the same findings. First, benchmarking effects cannot account for the behavior of HF managers, who follow absolute-returns strategies and thus faced no explicit performance concerns relative to bubble stocks. Second, for MF managers who faced this type of performance concerns, benchmarking models predict overinvestment, rather than underinvestment as Massa and Patgiri (2009) find, in benchmark stocks.

For very high levels of overvaluation, fee incentives can still induce over-investment in the stock through the exacerbation of absolute performance concerns. Indeed, Figs. 1(b) and 1(c) show that $A P H$ can turn positive and large for severe levels of overvaluation. The effect arises because, in these circumstances, the normal position is short and its magnitude increasing in the severity of the mispricing. The contrarian demand $A P H$ is then positive and can induce an overinvestment in the stock for these levels of overvaluation. Because, as mentioned above, APH is unrelated to benchmarking effects, it affects both types of managers equally. Therefore, it can lead to over-investment in the stock regardless of whether it belongs or not to the benchmark.

\subsubsection{Interim Trading in a Mispriced Stock}

The previous section illustrates the static response of a money manager to different levels of stock mispricing. Throughout the investment period, past values of stock mispricing affect the current value of the fund and benchmark portfolios, and hence the fund's relative performance.

\footnotetext{
${ }^{24}$ In the U.S, mutual funds are forbidden to charge asymmetric performance fees that reward outperformance but do not penalize underperformance. By contrast, this type of fees is commonplace among hedge funds (see Appendix B).
} 
We next study how the interaction between current mispricing and fund relative performance impacts the manager's interim $(0<t<T)$ trading. To do so, we take advantage of a novel feature of our setting, namely the flexibility to accommodate non-zero correlation between the two variables. ${ }^{25}$
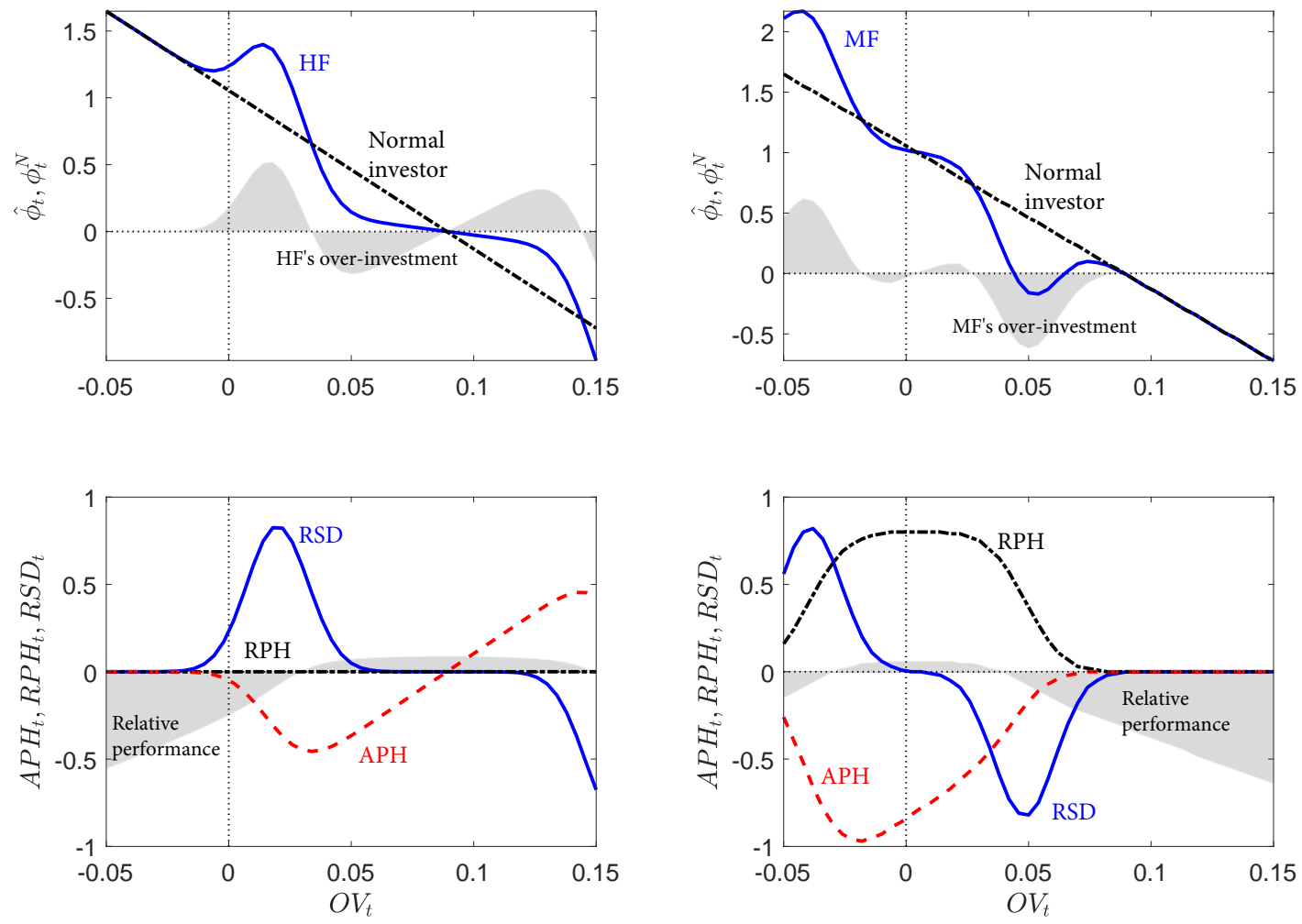

(a) $\operatorname{HF}\left(\phi^{Y}=0\right)$

(b) $\operatorname{MF}\left(\phi^{Y}=1\right)$

Figure 2: Managers' Interim Investment in a Mispriced Stock

This figure plots managers' and normal investments in the stock at different mispricing states $O V_{t}$, for $t=0.5 T$. Panel 2(a) illustrates the case of a hedge fund (HF) manager, while Panel 2(b) illustrates the case of a mutual fund (MF) manager subject to convex incentives. The top row across the two panels plots the manager's overall allocation $\hat{\phi}_{t}$ (solid blue line), the normal allocation $\phi_{t}^{N}$ (dash-and-dot black line), and the manager's over-investment $\hat{\phi}_{t}-\phi_{t}^{N}$ (shaded gray area) in the stock. The bottom row across the two panels plots the $R S D_{t}$ (solid blue line), $A P H_{t}$ (dashed red line) and $R P H_{t}$ (dash-and-dot black line) allocations, along with the manager's relative performance $\left(\hat{W}_{t}-\zeta Y_{T}\right) /\left(\zeta Y_{T}\right)$ (shaded gray area). The figure assumes an initial overvaluation $O V_{0}$ of $4 \%$. Parameter values are as in Figure 1.

Figure 2 illustrates the managers' optimal investment policies at $t=0.5 T$, as characterized

${ }^{25}$ In a single-risky asset setup, the time- $t$ correlation coefficient between changes in the manager's relative performance, $\hat{X}_{t} \equiv \hat{R}_{t} /\left(\bar{\zeta} R_{t}^{Y}\right)$, and changes in overvaluation, $O V_{t}$, is: $\operatorname{corr}_{t}\left(d \hat{X}_{t} / \hat{X}_{t}, d O V_{t}\right)=\left(\hat{\phi}_{t}-\phi^{Y}\right) /\left|\hat{\phi}_{t}-\phi^{Y}\right|$. Hence, $\operatorname{corr}_{t}\left(d \hat{X}_{t} / \hat{X}_{t}, d O V_{t}\right)$ is state dependent and equals either -1 or 1 , depending on whether the manager's portfolio at a particular state under- or overweights the stock relative to the benchmark. Unconditionally, however, this correlation is not perfect. In Section 3 we extend our analysis to more general values of the conditional correlation between these two variables. 
by Proposition 1, as a function of the time-t stock mispricing $O V_{t}$. For comparison purposes, the plots correspond to the HF and MF managers of Figure 1, identical except for the type of benchmark. To best highlight the associated implications, the figure assumes an initial scenario of relatively high stock overvaluation. ${ }^{26}$ Similarly to their initial positions, the managers' interim portfolios can substantially deviate from the normal policy (as illustrated by shaded area in the top two figures) for different overvaluation levels. However, these levels are now associated with different interim fund excess returns. Under convex incentives, changes in overvaluation that improve the managers' relative standing increase the $A P H_{t}$ and $R P H_{t}$ demands, as absolute and relative performance concerns intensify in the outperformance region. Hence, the interaction of mispricing and relative performance (depicted by the shaded area in the bottom two figures) leads to highly non-linear patterns in these demands. ${ }^{27}$ Along with the shifts in the gambling demand $R S D_{t}$, these effects give rise to several novel features.

First, a manager with no relative performance concerns with respect to an overvalued stock (e.g., the HF manager of Panel 2(a)) can increase the allocation to the stock as overpricing worsens, even when the normal portfolio reduces it. This is the case of the HF manager of Fig. 2 in the initial phases of overvaluation. According to Fig. 2(a), this behavior is driven exclusively by the gambling demand $R S D_{t}$, which invests an increasingly higher fraction of wealth in the stock as the manager seeks to catch up with the risk-free benchmark. Indeed, a surge in overvaluation that improves the relative performance of an underperforming manager exacerbates her risk seeking behavior. When the stock is underrepresented in the benchmark relative to the normal portfolio, the manager takes an even greater gamble on a future scenario of rising stock overvaluation. This demand more than offsets the negative and decreasing $A P H_{t}$ demand over

\footnotetext{
${ }^{26}$ Specifically, the plots correspond to an initial stock overvaluation $O V_{0}$ of $4 \%$ corresponding, given the parameter values we use in this Section (see footnote 23), to two standard deviations above $0 \%$. In this scenario, the HF manager of Fig. 1 initially overweights the stock by $3.8 \%$ whereas the MF manager underweights it by $21.4 \%$.

27 Although the extent of overvaluation is perfectly (negatively) correlated with the stock return in the singleasset setup of this section (this is in general not true under the multi-asset setting of Section 3), the relative performance of a manager displays a non-monotonic relation with the extent of mispricing. Indeed, relative performance first rises with $O V_{t}$, and then falls when $O V_{t}$ increases to very high levels in the bottom figures of Panels 2(a) and 2(b). This pattern stands in contrast with prior settings with a constant investment opportunity set (e.g., Basak, Pavlova, and Shapiro (2007)), in which relative performance maps one-to one onto (and is perfectly correlated with) the stock return.
} 
the same overvaluation range. The result is consistent with the "positive-feedback" trading of many hedge funds during certain phases of the tech bubble documented by Brunnermeier and Nagel (2004). Fig. 2(b) shows that, in stark contrast, the same effect can lead a manager with relative performance concerns (i.e., the MF manager) to sell an overvalued stock with positive risk premium, to which the normal policy allocates a positive weight.

Second, some deviations from the standard policy are exacerbated over the investment period. For instance, a manager whose benchmark excludes the stock (the HF manager) can overweight it more than an investor oblivious to the asset overpricing, who allocates a fixed weight $\phi=1$ to the stock. ${ }^{28}$ From the bottom figure in Panel 2(a), it is evident that this behavior responds to the hump in the manager's gambling demand $R S D_{t}$ in a neighborhood of the performance hurdle $\zeta Y_{t}=\zeta Y_{0} \exp (r t)$. This effect could explain the weight of highly overpriced technology stocks, larger in the portfolio of hedge funds than in the market portfolio, as documented by Brunnermeier and Nagel (2004).

Third, increases in overvaluation that lead to outperformance induce the HF manager to also increase the $A P H_{t}$ demand, as shown in Fig. 2(a). This effect significantly curbs overall selling of the stock relative to the normal policy for a range of severe overvaluation levels, despite the absence of short-sale restrictions in the model. This result agrees with the decline in short interest in NASDAQ stocks during the tech bubble documented by Lamont and Stein (2004), at a time when short-sale constraints on most tech stocks were unlikely to bind. As we show in the Internet Appendix C, these incentive-based limits to short-selling can hamper the role of sophisticated investors in stabilizing the stock market in the same fashion that explicit short-sale constraints limit pessimistic investors' trading against overvaluation in models of disagreement (see, e.g., Hong and Stein (2007)).

\footnotetext{
${ }^{28}$ This investor's portfolio corresponds to $\alpha=v_{0} \equiv 0$ in (12) and, given the assumption $\tilde{\mu}_{t}-r=\gamma \sigma_{t}^{2}$, represents an all-stock portfolio.
} 


\section{Multiple Assets and Abnormal Returns}

So far, we have presented results for the case of a single-risky asset. In this setting, the stock performs multiple functions; namely, it (i) captures the extent of mispricing, (ii) conveys the state of nature, and (iii) represents the total risk exposure of an investor as well as the only available risk premium in the economy. ${ }^{29}$ To disentangle the effects of these different dimensions on the trading of the manager, in this section we solve for the optimal portfolio of a manager who invests in multiple risky assets. Each asset is potentially subject to stochastic mispricing and, in general, does not fully summarize the state of the economy. In addition, mispriced assets might feature either independent or correlated abnormal expected returns, which in turn might exhibit zero, positive or negative correlation with other assets' observed returns.

\subsection{Multi-Asset Financial Markets}

Our framework follows Sotes-Paladino (2017), who generalizes the single-security market of Section 2.1 to an arbitrary number of securities. Specifically, we now assume $n+1 \geq 2$ risky assets with prices $S_{i}$ and fundamental values $F_{i}$, for $i=0,1, \ldots, n$. Let $r_{i, t}^{S} \equiv d \log \left(S_{i, t}\right)$ and $r_{i, t}^{F} \equiv d \log \left(F_{i, t}\right)$ denote, respectively, the growth in log stock price and fundamental value of asset $i$. The asset's mean return $E_{t}\left[r_{i, t}^{S}\right] / d t$ is the sum of the "fair" (or "normal") expected return $\tilde{r}_{i, t} \equiv E_{t}\left[r_{i, t}^{F}\right] / d t$, and a possibly nonzero "abnormal" expected return $\alpha_{i, t}$ (the asset's "alpha"). Letting the vector of Brownian motion processes $B=\left(B_{0}, B_{1}, \ldots, B_{n}\right)^{\prime}$ summarize the $n+1$ sources of risk in the economy, the $(\log )$ stock prices and fundamental values follow: ${ }^{30}$

$$
\begin{aligned}
& r_{i, t}^{S}=\left(\tilde{r}_{i, t}+\alpha_{i, t}\right) d t+\sigma_{i, t} d B_{t}, \\
& r_{i, t}^{F}=\tilde{r}_{i, t} d t+\delta_{i} d B_{t}
\end{aligned}
$$

for the (row) vector of return volatility processes $\sigma_{i, t} \equiv\left(\sigma_{i 0, t}, \sigma_{i 1, t}, \ldots, \sigma_{i n, t}\right)$, as specified below, and the (row) vector of constant fundamental volatility $\delta_{i}=\left(\delta_{i 0}, \delta_{i 1}, \ldots, \delta_{i n}\right)$. We denote $\Sigma_{t}=$

\footnotetext{
${ }^{29}$ We thank a referee for this observation.

${ }^{30}$ Henceforth, $a_{i}$ and $a^{j}$ denote, respectively, the $i$-th row and the $j$-th column of the matrix $A . I[X]$ denotes the diagonal matrix with vector $X$ in the diagonal.
} 
$\left(\sigma_{0, t}, \sigma_{1, t}, \ldots, \sigma_{n, t}\right)^{\prime}$ and $\Delta=\left(\delta_{0}, \delta_{1}, \ldots, \delta_{n}\right)^{\prime}$ the return and fundamental volatility matrices, which we assume are non-singular.

Following the empirical and theoretical literature (see references in Section 2.1), we let mispricing be stochastic. Given the initial vector $\alpha_{0}=\left(\alpha_{0,0}, \alpha_{1,0}, \ldots, \alpha_{n, 0},\right)^{\prime}$ and a symmetric positive semi-definite matrix $V_{0}$, the vector of abnormal expected returns $\alpha_{t}$ follows a vector autoregressive process of order one, $\operatorname{VAR}(1)$ :

$$
\begin{aligned}
& d \alpha_{t}=-2 \Delta V_{t} \Delta^{-1} \alpha_{t} d t-\Sigma_{t} V_{t} d B_{t}, \\
& d V_{t}=-V_{t}^{2} d t
\end{aligned}
$$

Under appropriate choice of the matrix $2 \Delta V_{t} \Delta^{-1}$ (e.g., diagonal with elements in the unit interval $[0,1)),(18)$ represents asset alphas that mean revert to zero over time. The matrix of alpha volatility $\Sigma_{t} V_{t}$ reflects total return volatility $\Sigma_{t}$, as well as the mispricing risk $V_{t}$ that magnifies $\Sigma_{t}$ relative to fundamental volatility $\Delta$ :

$$
\Sigma_{t}=\Delta\left(I+\tau V_{t}\right)
$$

Under these assumptions, we show in Appendix A the existence of a vector process $F$ such that the levels of asset prices and fundamental values are related via:

$$
\begin{aligned}
S_{i, t} & =F_{i, t} \exp \left(-\delta_{i} \Sigma_{t}^{-1} \alpha_{t} \tau\right), \\
& =F_{i, t} \exp \left(O V_{i, t}\right),
\end{aligned}
$$

where $O V_{i, t} \equiv-\delta_{i} \Sigma_{t}^{-1} \alpha_{t} \tau$ now represents the extent of asset overvaluation $\left(O V_{i, t}>0\right)$ or undervaluation $\left(O V_{i, t}<0\right)$ of asset $i=0,1, \ldots, n$. A negative (respectively, positive) alpha for asset $i$ maps one-to-one onto overvaluation (undervaluation) $O V_{i}$ of asset $i$ under appropriate parameterizations of $\Delta$ and $\Sigma_{t}$. In addition, the extent of over- or undervaluation is proportional to the "multivariate appraisal ratio" $\Sigma_{t}^{-1} \alpha_{t}$, and converges to zero no later than $t=T$. Eqs. (16), (17) and (21) imply that, for initial value $O V_{i, 0}=-\delta_{i} \Sigma_{0}^{-1} \alpha_{0} T$, the alpha of asset $i$ also 
determines its expected overvaluation dynamics $E_{t}\left[d O V_{i, t}\right]$ over the next instant $d t:^{31}$

$$
d O V_{i, t}=r_{i, t}^{S}-r_{i, t}^{F}=\alpha_{i, t} d t+\tau \delta_{i} V_{t} d B_{t}
$$

In principle, under multiple sources of risk, a manager's trading might depend on whether the asset's returns, or its mispricing, are subject to systematic or idiosyncratic risks. To examine this issue, and without loss of generality, we let $B_{0}$ be a source of systematic risk and $B_{1}, \ldots, B_{n}$ be $n$ sources of idiosyncratic risk. We further identify asset $i=0$ with a well-diversified, "market" portfolio, such that its fundamental value $F_{0}$ and abnormal expected return $\alpha_{0}$ are subject to systematic risk only. ${ }^{32}$ We let the fundamental value $F_{i}$ and abnormal expected returns $\alpha_{i}$ of the remaining $n$ risky assets $i=1, \ldots, n$ be potentially subject to both systematic and idiosyncratic risks. Under these assumptions, each asset's mean fair rate of return in excess of the risk-free rate, $\tilde{r}_{i, t}+1 / 2 \sigma_{i, t} \sigma_{i, t}^{\prime}-r$, is the compensation for the asset's exposure to systematic risk, $\gamma \sigma_{0, t} \sigma_{i, t}^{\prime}$. We denote $\tilde{\mu}_{i, t}=r+\gamma \sigma_{0, t} \sigma_{i, t}^{\prime}$ and $\mu_{i, t} \equiv \tilde{\mu}_{i, t}+\alpha_{i, t}$, respectively, the resulting fair and observed mean rates of return on the asset.

\subsection{Optimal Investment Strategy with Multiple Assets}

Markets completeness $(n+1$ risky asset prices $S$ driven by $n+1$ independent Brownian motions $B)$, along with no arbitrage, imply that the manager sees financial markets as driven by a unique state-price deflator (SPD)

$$
\pi_{t}=\exp \left\{-r t-\frac{1}{2} \int_{0}^{t}\left\|\eta_{s}\right\|^{2} d s-\int_{0}^{t} \eta_{s}^{\prime} d B_{s}\right\}
$$

\footnotetext{
31 Note that, for $\tau>0, V_{t}=\mathbf{0}_{n+1}$ (where $\mathbf{0}_{n+1}$ is the $(n+1) \times(n+1)$ zero matrix) implies no random fluctuations in $O V_{t}=\left(O V_{0, t}, O V_{1, t}, \ldots, O V_{n, t}\right)^{\prime}$. In this sense, the matrix $V_{t}$ reflects pure "mispricing risk."

${ }^{32}$ Letting $\alpha_{0}$ differ from zero allows for pricing errors in the market portfolio. This assumption is in line with Dumas, Kurshev, and Uppal (2009) and Brennan and Wang (2010), and generalizes the continuing cointegrated price framework in Liu and Timmermann (2013), who assume zero mispricing for the market portfolio.
} 
where $\eta=\Sigma^{-1}(\mu-r \mathbf{1})$ is the unique vector of market prices of risk, $\mathbf{1}$ is the $(n+1)$-dimensional unit vector, and $\|\cdot\|$ denotes the Euclidean norm in $\mathbb{R}^{n+1} \cdot 33$ Since $\mu=\tilde{\mu}+\alpha$,

$$
\eta_{t}=\tilde{\eta}_{t}+\kappa_{t}
$$

i.e., $\eta$ consists of the sum of the fair (no-mispricing) market prices of risk vector $\tilde{\eta}_{t} \equiv \Sigma_{t}^{-1}\left(\tilde{\mu}_{t}-\right.$ $r \mathbf{1})$, and the multivariate appraisal ratio $\kappa_{t} \equiv \Sigma_{t}^{-1} \alpha_{t}$.

The manager chooses fractions $\phi_{t}=\left(\phi_{0, t}, \phi_{1, t}, \ldots, \phi_{n, t}\right)^{\prime}$ of wealth $W_{t}$ to allocate in the risky assets $i=0,1, \ldots, n$, with the remaining fraction $1-\left(\phi_{t}\right)^{\prime} 1$ in the risk-less asset. The benchmark is the value $Y_{t}$ of a portfolio investing fixed fractions $\phi^{Y}=\left(\phi_{0}^{Y}, \phi_{1}^{Y}, \ldots, \phi_{n}^{Y}\right)^{\prime}$, with $\phi_{i}^{Y} \in[0,1]$, in the risky assets $i=0,1, \ldots, n$, with the rest $1-\left(\phi^{Y}\right)^{\prime} \mathbf{1}$ invested in the risk-less asset.

The manager solves the same portfolio problem (10) as in the single-risky asset case, with self-financing constraint and benchmark processes:

$$
\begin{gathered}
d W_{t}=W_{t}\left(r+\left(\phi_{t}\right)^{\prime}\left(\mu_{t}-r \mathbf{1}\right)\right) d t+W_{t}\left(\phi_{t}\right)^{\prime} \Sigma_{t} d B_{t}, \\
d Y_{t}=Y_{t}\left(r+\left(\phi^{Y}\right)^{\prime}\left(\mu_{t}-r \mathbf{1}\right)\right) d t+Y_{t}\left(\phi^{Y}\right)^{\prime} \Sigma_{t}, d B_{t},
\end{gathered}
$$

for initial values $W_{0}=w_{0}$ and $Y_{0}=y_{0}$.

We emphasize that the financial markets assumed in this section (as in Section 2.1) imply that the manager faces a stochastic investment opportunity set. For the type of kinked incentives we consider, prior studies have regarded optimal dynamic policies under a stochastic investment opportunity set as analytically intractable. ${ }^{34}$ However, the particular version we adopt allows us to characterize the optimal policy explicitly.

We first present the normal policy, that is, the optimal policy in the absence of convex incentives. Letting $\phi_{t}^{N}=\left(\phi_{0, t}^{N}, \phi_{1, t}^{N}, \ldots, \phi_{n, t}^{N}\right)^{\prime}$ denote the vector of fractions of wealth $W_{t}$ allocated to the risky assets $i=0,1, \ldots, n$ (with the balance $1-\left(\phi_{t}\right)^{\prime} \mathbf{1}$ in the risk-less asset) in the normal

\footnotetext{
${ }^{33}$ Our assumption that $\Delta$ is non-singular, along with our specification (20) imply that $\Sigma_{t}$ is non-singular for all $t \in[0, T]$.

${ }^{34}$ See, e.g., footnote 5 in Basak and Makarov (2014).
} 
portfolio, Sotes-Paladino (2017) characterizes $\phi_{t}^{N}$ as:

$$
\begin{aligned}
\phi_{t}^{N} & =\frac{1}{\gamma}\left(\Sigma_{t}^{-1}\right)^{\prime}\left(I+\tau V_{t}\right)\left(I+\frac{\tau}{\gamma} V_{t}\right)^{-1} \eta_{t} \\
& =\frac{1}{\gamma}\left(\Sigma_{t}^{-1}\right)^{\prime}\left(I+\tau V_{t}\right)\left(I+\frac{\tau}{\gamma} V_{t}\right)^{-1} \Sigma_{t}^{-1}\left(\tilde{\mu}_{t}-r \mathbf{1}+\alpha_{t}\right)
\end{aligned}
$$

To characterize the optimal portfolio in the presence of convex incentives, we introduce the following additional notation. Let $P$ be the orthogonal matrix such that the initial mispricing risk matrix can be diagonalized as:

$$
V_{0}=P^{\prime} I\left[d_{0}\right] P
$$

where $d_{0}=\left(d_{0,0}, d_{1,0}, \ldots, d_{n, 0}\right)^{\prime} \in \mathbb{R}^{n+1}$ is the vector of real, non-negative eigenvalues of $V_{0} \cdot{ }^{35}$ It can be shown that

$$
V_{t}=P^{\prime} I\left[d_{t}\right] P
$$

where $d_{t}=\left(d_{0, t}, d_{1, t}, \ldots, d_{n, t}\right)^{\prime} \in \mathbb{R}^{n+1}$ is the vector of eigenvalues of $V_{t}$, and for $i=0,1, \ldots, n$ :

$$
d_{i, t}=\frac{d_{i, 0}}{1+d_{i, 0} t}
$$

For a $(n+1) \times(n+1)$-dimensional matrix $X$ and a $(n+1)$-dimensional vector $x$, we denote $\bar{X}^{\prime}$ and $\bar{x}$, respectively, the rotated matrix $\bar{X}^{\prime} \equiv P X^{\prime}$ and vector $\bar{x} \equiv P x$. We present an analytical expression for the manager's portfolio under multi-asset mispricing in the following:

Proposition 2. For $t \in[0, T]$, the manager's time-t vector of optimal holdings of the risky assets $\hat{\phi}_{t}$ is:

$$
\begin{aligned}
\hat{\phi}_{t}= & \phi_{t}^{N}-\left[\sum_{i=1}^{2} \frac{\hat{W}_{i, t}}{\hat{W}_{t}}\left(1-\frac{\gamma}{\gamma_{i}}\right)\left(\Sigma_{t}^{-1}\right)^{\prime}\left(I+\frac{\tau}{\gamma_{i}} V_{t}\right)^{-1} \Sigma_{t}^{\prime}\right] \phi_{t}^{N}+\left[\sum_{i=1}^{2} \frac{\hat{W}_{i, t}}{\hat{W}_{t}}\left(1-\frac{\gamma}{\gamma_{i}}\right)\right] \phi^{Y} \\
& +\sum_{i=1}^{2} \frac{\hat{W}_{i, t}}{\hat{W}_{t}} \frac{\Phi_{i}\left(t, \phi_{t}^{N}-\phi^{Y}\right)}{\Pi_{i, t}}
\end{aligned}
$$

${ }^{35}$ Since by assumption $V_{0}$ is symmetric, there exists a $(n+1) \times(n+1)$ matrix $P$ such that $P^{\prime} P=P P^{\prime}=I$ (i.e., $P^{-1}=P^{\prime}$ ) and $(27)$ holds for the real-valued vector of eigenvalues $d_{0}$. Since $V_{0}$ is positive semi-definite by assumption, the elements of $d_{0}$ are non-negative. 
and the optimal level of assets under management is given by $\hat{W}_{t}=\hat{W}_{1, t}+\hat{W}_{2, t}$, where for $i=1,2, \hat{W}_{i, t} \equiv \pi_{t}^{-1} E_{t}\left[\pi_{T} \hat{W}_{i, T}\right]$ is characterized in Appendix A,

$$
\begin{aligned}
& \Phi_{i}\left(t, \phi_{t}^{N}-\phi^{Y}\right) \equiv-\frac{1}{\Pi_{i, t} \sqrt{\tau}}\left(\bar{\Sigma}_{t}^{-1}\right)^{\prime}\left(I+\tau I\left[d_{t}\right]\right)^{\frac{1}{2}}\left(I+\frac{\tau}{\gamma_{i}} I\left[d_{t}\right]\right)^{-\frac{1}{2}} \frac{\partial \Pi_{i, t}}{\partial A_{i, t}} \\
& \Pi_{i, t} \equiv \operatorname{Prob}\left(\overline{\mathcal{R}}_{i, t}\right)=\int_{\overline{\mathcal{R}}_{i, t}} \prod_{j=0}^{n} n\left(z_{j}\right) d z_{0} d z_{1} \ldots d z_{n} \\
& \overline{\mathcal{R}}_{i, t} \equiv\left\{\begin{array}{l}
\left\|\left(I+\frac{\tau}{\gamma_{1}} I\left[d_{t}\right]\right)^{-\frac{1}{2}}\left(\tau I\left[d_{t}\right]\right)^{\frac{1}{2}}\left(z-A_{1, t}\right)\right\|>\Gamma, \quad i=1 \\
\left\|\left(I+\frac{\tau}{\gamma_{2}} I\left[d_{t}\right]\right)^{-\frac{1}{2}}\left(\tau I\left[d_{t}\right]\right)^{\frac{1}{2}}\left(z-A_{2, t}\right)\right\| \leq \Gamma, \quad i=2
\end{array}\right. \\
& A_{i, t} \equiv \gamma\left(I+\frac{\tau}{\gamma_{i}} I\left[d_{t}\right]\right)^{-\frac{1}{2}}\left(I+\tau I\left[d_{t}\right]\right)^{\frac{1}{2}}\left(\tau^{\frac{1}{2}} I\left[d_{t}\right]\right)^{-1}\left(I+\frac{\tau}{\gamma} I\left[d_{t}\right]\right) \bar{\Delta}^{\prime}\left(\phi_{t}^{N}-\phi^{Y}\right)
\end{aligned}
$$

$z \equiv\left(z_{0}, z_{1}, \ldots, z_{n}\right)^{\prime}, n(\cdot)$ is as in Proposition $1, \lambda_{M}>0$ solves $\hat{W}_{0}=w_{0}$, and $\Gamma \geq 0$ is as in Appendix A.

The optimal multi-asset portfolio (30) keeps the structure of its single-asset counterpart (13). In particular, the incentives (9) induce a wedge between the investment policies of the money manager and the normal investor, which represents the manager's over- or underinvestment in each asset. This wedge equals the sum of the absolute performance hedge $A P H_{t} \equiv$ $-\sum_{i=1}^{2}\left[\hat{W}_{i, t} / \hat{W}_{t}\left(1-\gamma / \gamma_{i}\right)\left(\Sigma_{t}^{-1}\right)^{\prime}\left(I+\tau / \gamma_{i} V_{t}\right)^{-1} \Sigma_{t}^{\prime}\right] \phi_{t}^{N}$, the relative performance hedge $R P H_{t} \equiv$ $\sum_{i=1}^{2}\left[\hat{W}_{i, t} / \hat{W}_{t}\left(1-\gamma / \gamma_{i}\right)\right] \phi^{Y}$ and the risk-shifting demand $R S D_{t} \equiv \sum_{i=1}^{2} \hat{W}_{i, t} / \hat{W}_{t} \Phi_{i}\left(t, \phi_{t}^{N}-\right.$ $\left.\phi^{Y}\right) / \Pi_{i, t}$. Each of these demands has the same economic interpretation as in Section 2.3.2. In addition, the multi-asset formula (30) reveals that $A P H_{t}$ and $R P H_{t}$ maintain the relative weights of each stock in the normal and benchmark portfolios, respectively (with the opposite sign in the case of $A P H_{t}$ ). We next illustrate how these demands, and especially $R S D$, give rise to new results in the presence of correlated mispricing.

\subsection{Money Manager's Trading Under Multi-Asset Mispricing}

In financial markets with multiple assets subject to risky mispricing, an asset's abnormal expected return $\alpha_{i}$ can be exposed to systematic risk (as in Section 2), idiosyncratic risk, or both. Moreover, the asset's fair return $r_{i}^{F}$ can also be exposed to either or both sources of risk. Propo- 
sition 2 covers all these possibilities, for an arbitrary number of assets. However, to simplify the analysis we make the following assumptions. First, we set $n=1$, so that in addition to the market portfolio $(i=0)$, investors can trade in one risky asset $(i=1)$. Second, we assume that the fair return $r_{1}^{F}$ to asset 1 is exposed to idiosyncratic risk only.

As in our earlier analysis, we consider hedge fund (HF) and mutual fund (MF) managers. In addition, we distinguish between two types of MF managers depending on their benchmarks. One manager, running mutual fund "S," is benchmarked against the market index $\left(\phi^{Y}=(1,0)^{\prime}\right)$. The other manager, running mutual fund "I," is benchmarked against the "idiosyncratic" asset $1\left(\phi^{Y}=(0,1)^{\prime}\right)$.

\subsubsection{Introduction of Idiosyncratic Mispricing Risk}

An issue we can address with multiple risky assets is to what extent the trading patterns we find in Section 2 change with the availability alongside the market index of idiosyncratic investments. For instance, a manager that over-invests in an overvalued market index just to alter the overall portfolio risk in a desired direction could instead achieve the same goal by investing, if available, in a fairly priced idiosyncratic security. As we detail in Internet Appendix B, we can answer this question by assuming that the abnormal expected returns of assets 0 and 1 are uncorrelated, so that both the fair and abnormal returns to asset 1 are exposed to idiosyncratic risk only. Since under this assumption asset 1 commands no fair risk premium (i.e., no fair return in excess of the risk-free rate), we refer to it as a pure idiosyncratic investment.

We draw two main observations from our analysis in Internet Appendix B. First, the availability of fairly priced idiosyncratic investments does not change our results of Section 2.4.1. Since the expected excess return to asset 1 is zero, the HF, MF S manager - as defined aboveand the normal investor all allocate zero weight to this asset. They invest in the market index and the risk-free asset with the same trading patterns as in the single-stock case. By contrast, benchmarking effects lead the MF I manager to overweight the idiosyncratic investment for all levels of mispricing of the market index. Second, when the market index is fairly priced, all types of managers can overinvest in an overvalued idiosyncratic investment. When asset 1 is 
overvalued its risk premium is negative. In this case, absolute performance concerns can lead managers without benchmarking concerns relative to asset 1 (i.e., the HF and MF S managers) to overweight this asset in their portfolios, although the overall position in the asset remains negative. The effect takes place when the normal policy sells the overpriced stock short but absolute-performance considerations induce an overall smaller short position in the manager's portfolio.

\subsubsection{Effects of Correlated Mispricing}

In practice, exploiting temporary mispricing usually requires trading in assets with potentially correlated alphas. For instance, "convergence trade strategies" (e.g., "pairs trading") involve trading in pairs of assets whose abnormal expected returns are perfectly correlated (Liu and Timmermann, 2013). When this is the case, asset diversification and substitution considerations, both in isolation and through their interaction with incentive convexity and benchmarking effects, could have a non-negligible impact on the manager's optimal portfolio. We analyze this possibility in Figure 3, which plots the initial portfolio weights in assets 0 (the market index, top row) and 1 (bottom row), when abnormal expected returns are positively correlated across assets. In Panel 3(a) we vary the mispricing of asset 0 and keep the overpricing of asset 1 fixed, while in Panel 3(b) we keep the overpricing of asset 0 fixed and vary the mispricing of asset 1 . For brevity, we present results only for the case of positive correlation, but similar conclusions obtain under negative mispricing correlation. ${ }^{36}$

Correlated mispricing can magnify the effects we presented in previous sections and give rise to novel patterns. In particular, a manager can hold a long position in an overvalued, negativerisk premium asset that is not included in the benchmark. This is illustrated by the positive allocations of the HF and MF S managers of Panel 3(a) in the negative-risk premium, nonbenchmark asset 1 , for moderate or high overvaluation levels of the market index. The result is

\footnotetext{
${ }^{36}$ Our calibration of the fee incentive and market index parameters is as in Section 2.4. The assumed parameters imply a cross-asset correlation in overvaluation changes of 0.98 , a cross-asset return correlation of 0.18 , volatility of overvaluation changes of $2.5 \%$ for asset 0 and $2.9 \%$ for asset 1 , return volatilities of $15 \%$ for asset 0 and $18 \%$ for asset 1 , correlation between the return and the overvaluation changes of asset 0 (respectively, asset 1 ) of 0.85 (0.83), and a correlation between the return of asset 0 (asset 1 ) and the overvaluation changes of asset 1 (asset $0)$ of $0.71(0.69)$.
} 
striking when contrasted with the negative weight of the same asset in the normal portfolio, as it implies that the managers would instead short sell the asset if subject to standard incentives. The long positions of the MF I manager in the non-benchmark market index (Panel 3(a)), and of the MF S manager in the non-benchmark asset 1 (Panel 3(b)), for specific negative ranges of their respective risk premiums, provide additional examples of this behavior.
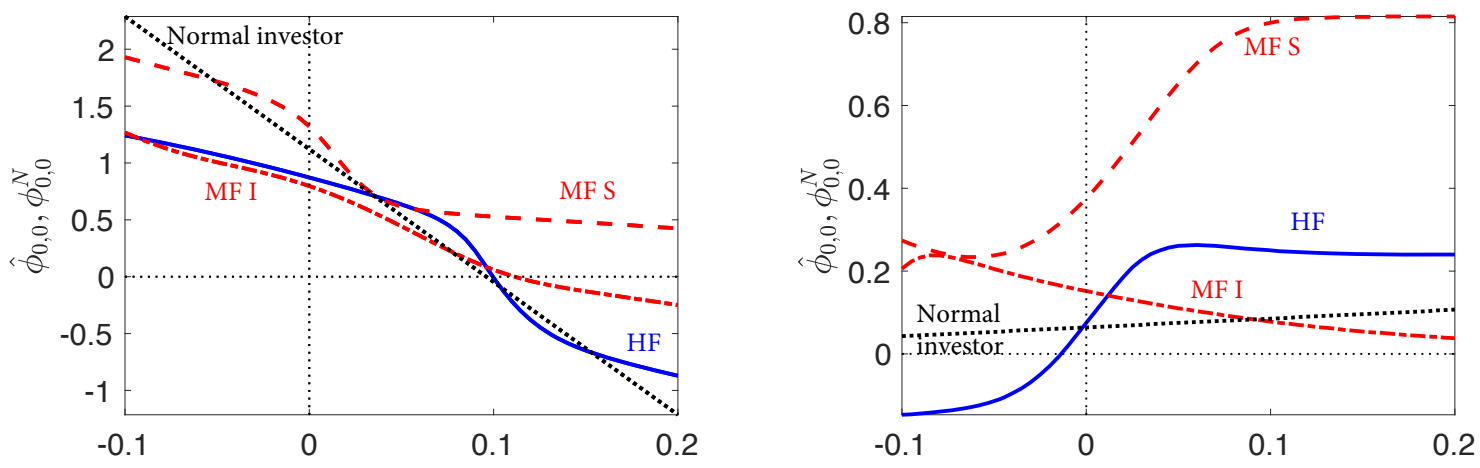

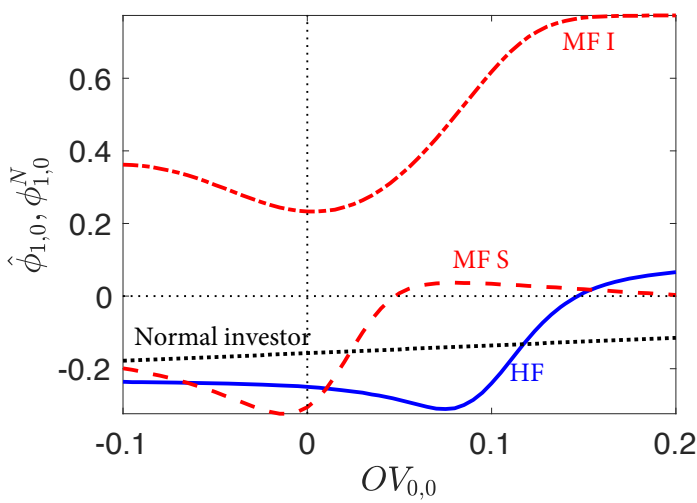

(a) Mispricing of Asset $0\left(O V_{1,0}=0.03\right)$

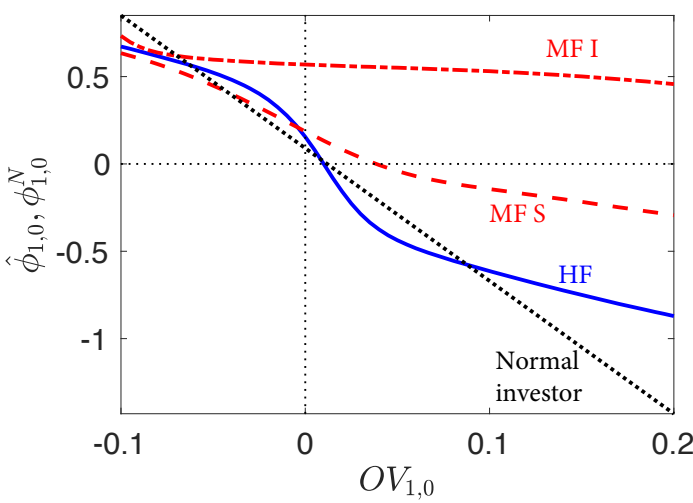

(b) Mispricing of Asset $1\left(O V_{0,0}=0.09\right)$

Figure 3: Initial Portfolio Weights in Assets with Correlated Abnormal Returns

This figure plots the money managers' investments in two risky assets at different initial mispricing states $O V_{0,0}$ (Panel 3(a)) and $O V_{1,0}($ Panel 3(b)). Across panels, we illustrate the initial portfolio weights allocated to asset 0 (top row) and asset 1 (bottom row) of the HF manager (solid blue line), MF S manager (dashed red line), MF I manager (dash-and-dot red line) and the normal investor (dotted black line). Parameter values are: $\psi_{1}=0, \psi_{2}=5, \bar{\zeta}=1, \gamma=5, T=1, r=1.5 \%, \delta_{0}=(0.129,0), \delta_{1}=(00.16), v_{0,0}=$ $(0.1502,0.1163), v_{1,0}=(0.1163,0.1406)$.

The result derives from the combination of cross-asset diversification (substitution) and convexity effects. When their mispricing is positively correlated and the relative overvaluation of asset 0 -i.e., the overvaluation of asset 0 in excess of the overvaluation of asset 1 -increases, one would expect, even in the absence of convex incentives, some substitution of asset 1 for asset 
0 in an investor's portfolio. By reallocating between the two assets, the investor can exploit the relative mispricing while limiting portfolio exposure to mispricing risk. Fig. 3(a) shows that risk aversion can prevent a normal investor from taking advantage of the relative mispricing aggressively, and that the substitution effect just translates into a small reduction in the short position in asset 1 .

By contrast, the managers' risk-shifting demand in response to convex incentives can exacerbate this substitution effect to the point of inducing a long position in this asset. This is shown in Figure 4, which decomposes the optimal portfolios of the HF and MF S managers of Fig. 3(a) into their $A P H, R P H$ and $R S D$ components. ${ }^{37}$ For comparison, we include the same components of the managers' portfolios under uncorrelated mispricing (as in Section 3.3.1). Consider the HF manager of the top row of Fig. 4, under the correlated mispricing scenario. When the risk premium of asset 0 is negative yet larger than the risk premium of asset 1 (consistent with $0.10<O V_{0,0}<0.13$ in the graph), the relative mispricing is too small to be exploited aggressively. The gambling demands for both assets have the same sign as the normal policy and lead to above-normal short selling of the two assets, as illustrated in Fig. 3(a). As the overvaluation of asset 0 worsens and its risk premium falls further relative to the risk premium of asset $1, R S D$ for asset 1 increases rapidly, and eventually turns positive and large. A similar effect explains the positive position of the MF S manager of the bottom row of Fig. 4 in the negative-risk premium, non-benchmark asset 1, for high enough overvaluation of the benchmark asset 0 . For both types of managers, the interaction of asset substitution and convexity effects is strong enough to induce positive demands for asset 1 only when mispricing is correlated. The result is surprising in light of the conclusions of Basak, Pavlova, and Shapiro (2007), as it implies that in this case the gambling demand can take the opposite sign of the excess holdings of the normal policy relative to the benchmark, negative for both HF and MF S managers.

The over-investment in an asset with negative risk premium illustrates how convex incentives can induce "bubble-riding" behavior. DeMarzo, Kaniel, and Kremer (2008) define a bubble

\footnotetext{
37 In Appendix C, we illustrate the importance of convexity versus pure benchmarking effects in inducing this result with an alternative approach, based on comparing benchmarked managers with and without $\left(\psi_{1}=\psi_{2}\right)$ convex incentives. We thank Andrea Buffa for suggesting this analysis.
} 

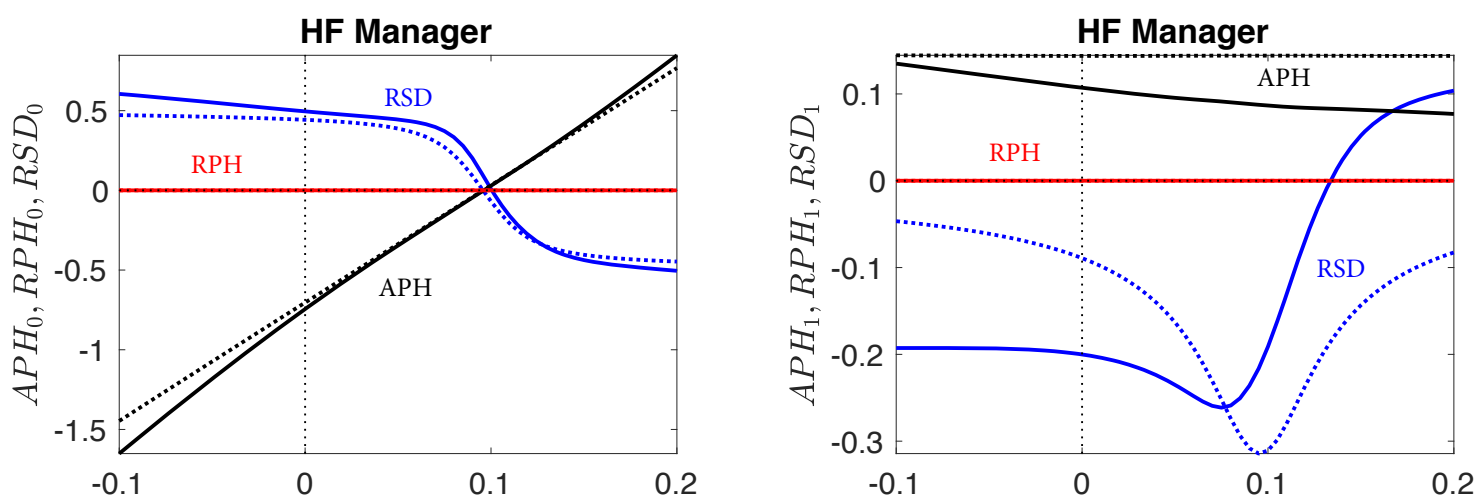

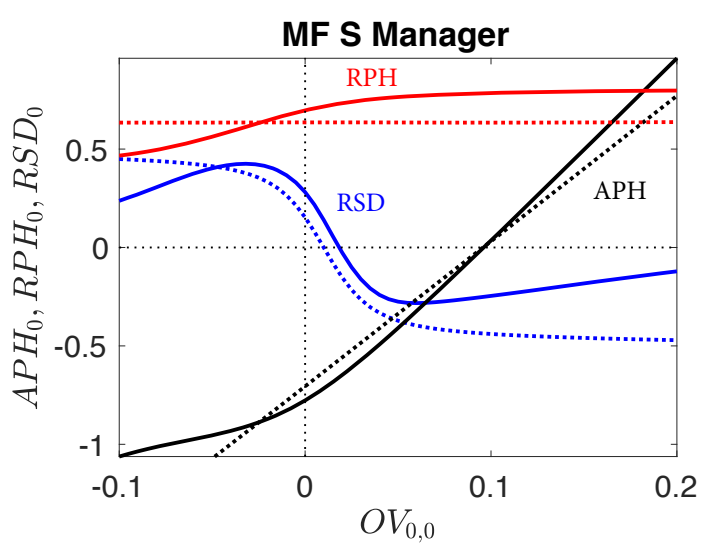

(a) Holdings of Asset 0

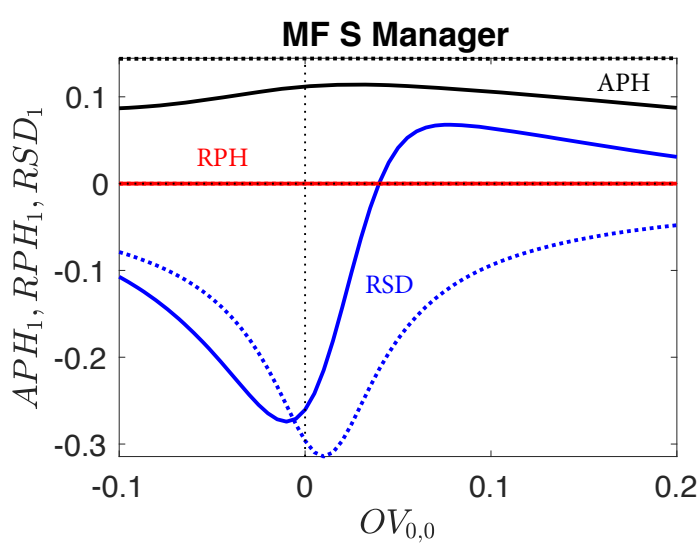

(b) Holdings of Asset 1

Figure 4: $A P H, R P H$ and $R S D$ components in Managers' Initial Portfolios

This figure plots, for different initial mispricing states $O V_{0,0}$ of assets 0 (keeping $O V_{1,0}=0.03$ ), the money managers' initial hedging demands for assets 0 (Panel 4(a)) and 1 (Panel 4(b)) in their optimal portfolios of Fig. 3(a). The blue, black and red lines represent, respectively, the $R S D, A P H$ and $R P H$ allocations of the HF (top row) and MF S (bottom row) managers. The solid lines correspond to the case of positive-mispricing correlation, with mispricing risk matrix $V_{0}$ as in Fig. 3. The dashed lines correspond to the case of zero-mispricing correlation, with mispricing risk matrix $V_{0}$ as in Fig. IA.2 of the Internet Appendix. The rest of the parameters are as in Fig. 3.

in an economy with risk-averse agents as a price that yields a negative risk premium for an asset whose cash flows have a nonnegative correlation with aggregate risk. The severe levels of overvaluation associated with negative risk premia in our examples fit this definition. When mispricing is deterministic (as in the constant investment opportunity set of prior literature or Internet Appendix A), or stochastic but uncorrelated across assets (sections 2 and 3.3.1), absolute performance concerns can lead managers to short sell a non-benchmark bubble asset less than a normal investor. However, they do not lead to a long position in this type of asset. ${ }^{38}$

\footnotetext{
${ }^{38}$ Even if the assets are fundamentally correlated, we verify in unreported results that under deterministic
} 
The reason is that, in these cases, the risk-shifting demand $R S D$ deviates from the benchmark in the same direction as the normal policy, leading to an overall negative position in a bubble asset. As our results in this section show, this effect does not generalize, and can actually be overturned, under stochastic and correlated mispricing.

\subsubsection{Empirical Implications}

Our results in this section have important empirical implications. First, they can rationalize the pairs trading strategy used by sophisticated investors such as hedge funds over short investment horizons. Liu and Timmermann (2013) show that the long-short portfolio that implements this strategy is generally suboptimal for risk-averse investors, and demonstrate that the optimal short-term trading in two negative-alpha (i.e., overvalued) assets under risk aversion consists of holding short positions in both assets. Our model shows that this result is overturned when we account for the convex incentives of risk-averse arbitrageurs.

Intuitively, the relative mispricing of two overvalued, potentially negative-risk premia assets creates the opportunity to make a profit by trading in the two (potentially non-benchmark) assets. Under positive and high correlation in the assets' abnormal expected returns, future mispricing risk can be hedged by taking opposite positions in the two assets - i.e., a long-short strategy. Following Liu and Timmermann (2013), for a standard (normal) risk-averse investor the strategy might be too risky over the short horizon considered in Section 3.3.2. Indeed, the normal portfolio in Panel 3(a) is dominated by mean-variance considerations, which depend on the instantaneous risk-return trade-off of the assets. Thus, a normal investor sells assets 0 and 1 short when both are overvalued and the overvaluation of asset 0 is large enough. By contrast, convex incentives exacerbate the intertemporal hedging demand of the manager, eventually offsetting the mean-variance component. The ensuing $R S D$ demands resembles the standard arbitrage strategy that finances the purchase of the relatively less expensive asset by short selling the more expensive asset. Benchmarking effects play no role in this result.

mispricing convex incentives alone - i.e., in the absence of benchmarking effects - cannot lead to a positive portfolio allocation in a negative-risk premium asset that a normal investor sells short. 
Second, our findings can rationalize further features of the behavior of some institutional investors during the tech bubble, as documented by the empirical literature. Griffin, Harris, Shu, and Topaloglu (2011) point to empirical evidence indicating that the overall market (as measured by, e.g., the S\&P500 index) was becoming overvalued by the late 1990s, and highlight the existence of positive mispricing correlation across indexes during this episode. ${ }^{39}$ The results in this section can explain as an optimal response to their incentives the net positive positions in bubble - i.e., negative-risk premium - assets of some institutional investors reported by these authors. The strategy of the MF S manager in our setup could also help explain the overinvestment in technology stocks by mutual fund managers benchmarked against a broad market index, as documented by Greenwood and Nagel (2009). They find that, during the bubble, younger mutual fund managers invested more in overvalued, non-benchmark (tech) stocks than their more experienced peers. At the same time, their benchmark tracking error was smaller. Arguing that these findings cannot be explained by either career concerns or convex payoff models, the authors regard their evidence as a puzzle in light of existing theories. According to our results, the documented higher flow convexity faced by young managers (Chevalier and Ellison, 1997) along with both the convexity in these managers' incentives and the risks and correlation in the mispricing they faced can explain this puzzle. ${ }^{40}$

\section{Convex Incentives and Equilibrium Mispricing}

We have shown that price-taking money managers with convex incentives might trade less aggressively against mispricing than if such incentives were absent. Our results also hold under more general settings, though. In particular, we show in the Internet Appendix C that our findings are robust to the mispricing dynamics that would arise in an equilibrium model in which

\footnotetext{
39 A further reason to validate the assumption of positive mispricing correlation during the technology bubble is given by Pastor and Veronesi (2009). They argue that when a new technology is exploited at small scale the firm's stock return displays purely idiosyncratic risk. As the technology scales up, the stock becomes part of the market portfolio and its returns exposed to systematic risk. At this stage, uncertainty about the technology can lead to bubble-like asset price dynamics, much as it was observed in the technology sector during the late 1990s.

40 In unreported results, we verify that more convex incentives lead to larger risk-shifting and relativeperformance hedging demands. The first effect can explain the larger position of young managers in overvalued non-benchmark stocks, whereas the second can account for their lower benchmark tracking error.
} 
money managers have price impact.

Our equilibrium framework follows closely the setting of Section 2. Investors trade continuously in a single risky asset (stock) and a risk-less asset over a finite investment horizon. To examine the price impact of managers with convex incentives when prices deviate from fundamental value, we assume that investors can be of either of two types: informed (sophisticated) managers and uninformed (unsophisticated) retail traders. Managers are informed because they observe the stock fundamentals. Retail traders neither observe fundamentals nor recognize that managers do. In this sense, they are "sentiment-driven" investors (as in, e.g., Dumas, Kurshev, and Uppal (2009)) who follow their own prior and learn the stock fundamentals over time, from price realizations. Given their inference errors, they can over- or under-weight the stock in their portfolios relative to otherwise identical investors who are as informed as the managersnormal investors. The trading of the retail investors can thus push stock prices above or below fundamental value, defined as the fair price prevailing under no mispricing or mispricing risk.

The effect of convex incentives on asset prices in general equilibrium can be inferred from our earlier analysis. Absent convex incentives, an informed (normal) investor always pushes prices towards fundamental values, reducing the extent of mispricing across economic states. When managers have convex incentives, their optimal strategy can exacerbate the overpricing of a non-benchmark stock relative to the case of no convex incentives. The distortion is unrelated to benchmarking effects induced by performance concerns relative to the stock. Instead, it is driven by the manager's hedging demand in response to risk-shifting and absolute performance considerations. The exacerbation of asset prices can become severe over the investment period, when the manager's risk-shifting demand can inflate the price of an overvalued asset even more than overoptimistic retail traders do.

\section{Conclusions}

An important puzzle of the last decades is why sophisticated investors have taken long positions in overpriced securities, as several studies document during the technology bubble of the late 1990s and early 2000s. We extend the literature on optimal trading of money managers with 
convex incentives (e.g., hedge fund and mutual fund managers) by including securities that feature stochastic and possibly correlated mispricing. In the model, managers are aware of the degree of mispricing. We derive and analyze, for the first time in the literature, their optimal portfolio in this setting. The demand for overpriced securities is affected by benchmarking effects, as studied extensively in previous literature, but also by risk-shifting and absolute performance considerations. The level of mispricing affects mostly the last two effects. We show that the interaction of these effects at various levels of mispricing, both before and after accounting for correlation in mispricing across assets, gives rise to several surprising results. We also show that, in equilibrium, the investment strategies of managers with convex incentives can exacerbate security mispricing.

Our rich setting provides a possible explanation for several puzzles raised by the empirical literature on institutional money management. For instance, we are able to rationalize the overinvestment of sophisticated investors, such as hedge funds, in overpriced securities that do not belong to their benchmarks. We also explain the aggressive underinvestment of similarly sophisticated investors in overvalued benchmarks securities. Furthermore, our findings help explain the long positions of mutual and hedge funds in negative-risk premium, "bubble" securities, even when they are not part of their benchmarks. Lastly, we rationalize "pairs trading" in two overvalued securities over short horizons, which prior studies show violate optimal investment rules in the absence of the type of incentives we consider. Overall, our findings demonstrate that, once realistic features of the managers' incentives are accounted for, rational behavior in response to these incentives can go a long way in explaining some apparently puzzling evidence. 


\section{References}

Abreu, D., and M. K. Brunnermeier, 2003, "Bubbles and crashes," Econometrica, 71, 173-204.

Allen, F., and G. Gorton, 1993, "Churning bubbles," Review of Economic Studies, 60, 813-836.

Basak, S., and D. Makarov, 2014, "Strategic Asset Allocation in Money Management," Journal of Finance, 69, 179-217.

Basak, S., and A. Pavlova, 2013, "Asset Prices and Institutional Investors," American Economic Review, 103, 1728-1758.

Basak, S., A. Pavlova, and A. Shapiro, 2007, "Optimal Asset Allocation and Risk Shifting in Money Management," Review of Financial Studies, 20, 1583-1621.

Brennan, M. J., and A. W. Wang, 2010, "The Mispricing Return Premium," Review of Financial Studies, 23, 3437-3468.

Brennan, M. J., and Y. Xia, 2001, "Assessing Asset Pricing Anomalies," Review of Financial Studies, 14, 905-945.

Brunnermeier, M. K., and S. Nagel, 2004, "Hedge Funds and the Technology Bubble," Journal of Finance, 59, 2013-2040.

Buffa, A., D. Vayanos, and P. Woolley, 2014, "Asset Management Contracts and Equilibrium Prices," Working Paper, Boston University and London School of Economics.

Buraschi, A., R. Kosowski, and W. Sritrakul, 2011, "Incentives and Endogenous Risk Taking: A Structural View of Hedge Funds Alphas," Working Paper, AFA 2012 Chicago Meetings Paper. Available at SSRN: http://ssrn.com/abstract=1785995 or http://dx.doi.org/10.2139/ssrn.1785995.

Chevalier, J., and G. Ellison, 1997, "Risk Taking by Mutual Funds as a Response to Incentives," Journal of Political Economy, 105, 1167-1200.

Cuoco, D., and R. Kaniel, 2011, "Equilibrium Prices in the presence of Delegated Portfolio Management," Journal of Financial Economics, 101, 264-296.

Dass, N., M. Massa, and R. Patgiri, 2008, "Mutual Funds and Bubbles: The Surprising Role of Contractual Incentives," Review of Financial Studies, 21, 51-99.

DeMarzo, P., R. Kaniel, and I. Kremer, 2008, "Relative Wealth Concerns and Financial Bubbles," Review of Financial Studies, 21, 19-50.

Drechsler, I., 2013, "Risk Choice under High-Water Marks," forthcoming, Review of Financial Studies. 
Dumas, B., A. Kurshev, and R. Uppal, 2009, "Equilibrium Portfolio Strategies in the Presence of Sentiment Risk and Excess Volatility,” Journal of Finance, 64, 579-629.

Farnsworth, H., and J. Taylor, 2006, "Evidence on the Compensation of Portfolio Managers," Journal of Financial Research, 29, 305-324.

Gatev, E., W. N. Goetzmann, and K. G. Rouwenhorst, 2006, "Pairs Trading: Performance of a Relative-Value Arbitrage Rule," Review of Financial Studies, 19, 797-827.

Greenwood, R., and S. Nagel, 2009, "Inexperienced Investors and Bubbles," Journal of Financial Economics, 93, 239-258.

Griffin, J. M., J. H. Harris, T. Shu, and S. Topaloglu, 2011, "Who Drove and Burst the Tech Bubble?," Journal of Finance, 66, 1251-90.

Hodder, J. E., and J. C. Jackwerth, 2007, "Incentive Contracts and Hedge Fund Management," Journal of Financial and Quantitative Analysis, 42, 811-826.

Holmen, M., M. Kirchler, and D. Kleinlercher, 2014, "Do option-like incentives induce overvaluation? Evidence from experimental asset markets," Journal of Economic Dynamics \&3 Control, 40, 179?194.

Hong, H., and J. C. Stein, 2007, "Disagreement and the Stock Market," Journal of Economic Perspectives, 18, 3-26.

Huang, J., K. D. Wei, and H. Yan, 2007, "Participation Costs and the Sensitivity of Fund Flows to Past Performance," Journal of Finance, 62, 1273-1311.

Ibert, M., R. Kaniel, S. V. Nieuwerburgh, and R. Vestman, 2017, "Are Mutual Fund Managers Paid For Investment Skill?," forthcoming, Review of Financial Studies.

Johnson, T. C., 2004, "Forecast Dispersion and the Cross Section of Expected Returns," Journal of Finance, 59, 1957-1978.

Kaniel, R., and P. Kondor, 2013, "The Delegated Lucas Tree," Review of Financial Studies, 26, 929-984.

Lamont, O., and J. Stein, 2004, "Aggregate Short Interest and Market Valuations," American Economic Review, 94, 29-32.

Liu, J., and F. A. Longstaff, 2004, "Losing Money on Arbitrage: Optimal Dynamic Portfolio Choice in Markets with Arbitrage Opportunities," Review of Financial Studies, 17, 611-641.

Liu, J., and A. Timmermann, 2013, "Optimal Convergence Trade Strategies," Review of Financial Studies, 26, 1048-1086.

Ma, L., Y. Tang, and J.-P. Gomez, 2015, "Portfolio Manager Compensation in the U.S. Mutual Fund Industry," Working Paper. 
Madhavan, A., and S. Smidt, 1993, "An Analysis of Changes in Specialist Inventories and Quotations," Journal of Finance, 48, 1595-1628.

Makarov, I., and G. Plantin, 2014, "Rewarding Trading Skills Without Inducing Gambling," forthcoming, Journal of Finance.

Malamud, S., and E. Petrov, 2014, "Portfolio Delegation and Market Efficiency," Working Paper, Swiss Finance Institute.

Massa, M., and R. Patgiri, 2009, "Incentives and Mutual Fund Performance: Higher Performance or Just Higher Risk Taking?," Review of Financial Studies, 22, 1777-1815.

Panageas, S., and M. M. Westerfield, 2009, "High-Water Marks: High Risk Appetites? Convex Compensation, Long Horizons, and Portfolio Choice," Journal of Finance, 64, 1-36.

Pastor, L., and P. Veronesi, 2009, "Technological Revolutions and Stock Prices," American Economic Review, 99, 1451-1483.

Poterba, J., and L. Summers, 1988, "Mean Reversion in Stock Prices," Journal of Financial Economics, 22, 27-59.

Sato, Y., 2009, "Ranking Concerns of Fund Managers and the Persistence of Bubbles," Working Paper, London School of Economics.

Shleifer, A., and R. W. Vishny, 1997, "The Limits of Arbitrage," Journal of Finance, 52, 35-55.

Sirri, E. R., and P. Tufano, 1998, "Costly Search and Mutual Fund Flows," Journal of Finance, $53,1589-1622$.

Sotes-Paladino, J. M., 2017, "Optimal Trading in Correlated Anomalies," Working Paper, The University of Melbourne.

Stein, J., 2009, "Sophisticated Investors and Market Efficiency," Journal of Finance, 64, 151748.

Vayanos, D., and P. Woolley, 2013, "An Institutional Theory of Momentum and Reversal," Review of Financial Studies, 26, 1087-1145.

Wang, J., 1993, "A Model of Intertemporal Asset Prices Under Asymmetric Information," Review of Economic Studies, 60, 249-282. 


\section{Appendix}

\section{A Proofs and Auxiliary Results}

Proof of Equations (6) and (21). We prove the multi-asset version Eq. (21), of which Eq. (6) is a particular case. To prove (21), it suffices to find a process $F_{i, t}$ that is a solution of the SDE (17) and show that $S_{i, t}$, as defined by (21), is a solution of the SDE (16). Define the asset $i$ 's fundamental value as

$$
F_{i, t}=D_{i, t} \exp \left\{\left(\delta_{i} \theta-r-\gamma \delta_{00} \sigma_{i, 0}+\frac{1}{2} \tau\left(\sigma_{i, t}-\delta_{i}\right) \delta_{i}^{\prime}\right) \tau\right\}, \quad t \in[0, T]
$$

where $\theta=\left(\theta_{0}, \theta_{1}, \ldots, \theta_{n}\right) \in \mathbb{R}^{n+1}, \gamma$ is a positive constant, and $D_{i, t}$ is a geometric Brownian motion process:

$$
d D_{i, t}=\delta_{i} \theta D_{i, t} d t+D_{i, t} \sum_{i=0}^{n} \delta_{i j} d B_{i, t}, \quad t \in[0, T],
$$

with initial value $D_{i, 0}=\bar{D}_{i}>0$.

To prove that $F_{i, t}$ that is a solution of the $\operatorname{SDE}(17)$, note that:

$$
\begin{aligned}
\log F_{i, t}= & \log D_{i, 0}+\left(\delta_{i} \theta-\frac{1}{2} \delta_{i} \delta_{i}^{\prime}\right) t+\delta_{i} B_{t} \\
& +\left(\delta_{i} \theta-r-\gamma \delta_{00} \delta_{i}\left(I+\tau V_{t}\right) e_{0}+\frac{1}{2} \tau \delta_{i} V_{t} \delta_{i}^{\prime}\right) \tau .
\end{aligned}
$$

Thus, $\log F_{i, t}$ is a twice continuously differentiable function of $t, B_{t}$. Applying Itô's rule, using the definition (20) and rearranging gives

$$
d \log F_{i, t}=\left(r+\gamma \sigma_{0, t} \sigma_{i, t}^{\prime}-\frac{1}{2} \sigma_{i, t} \sigma_{i, t}^{\prime}\right) d t+\delta_{i} d B_{t}
$$

Noting that the first term in the RHS of (A2) is the definition of $\tilde{r}_{i, t}$, we obtain (17).

To prove that $S_{i, t}$, as defined by (21), is a solution of the $\operatorname{SDE}(16)$, we note that:

$$
d \log S_{i, t}=d \log F_{i, t}-\delta_{i} d\left(\kappa_{t} \tau\right) .
$$

We obtain $d\left(\kappa_{t} \tau\right)$ by applying Itô's rule, first to $\kappa_{t}=\Sigma_{t}^{-1} \alpha_{t}$ :

$$
\begin{aligned}
d \kappa_{t} & =d\left(\Sigma_{t}^{-1}\right) \alpha_{t}+\Sigma_{t}^{-1} d \alpha_{t} \\
& =-V_{t} \kappa_{t} d t-V_{t} d B_{t},
\end{aligned}
$$

and then to $\kappa_{t} \tau$ :

$$
\begin{aligned}
d\left(\kappa_{t} \tau\right) & =d \kappa_{t} \tau+\kappa_{t} d \tau \\
& =-\left(I+\tau V_{t}\right) \kappa_{t} d t-\tau V_{t} d B_{t} .
\end{aligned}
$$


Substituting back into (A3) and using the definitions of $\Sigma_{t}$ and $\alpha_{t}$ gives (16). The proof holds for $n \geq 0$, so in particular it holds for $n=0$, which proves Eq. (6).

Proof of Proposition 1. Included at the end of the proof of Proposition 2 below.

Proof of Proposition 2. We start by characterizing the manager's optimal policy in terms of end-of-period AUM:

Proposition A1. The manager's time-T optimal state-contingent value of the assets under management is $\hat{W}_{T}=\hat{W}_{1, T}+\hat{W}_{2, T}$, where for $i=1,2$ :

$$
\begin{aligned}
& \hat{W}_{i, T}=\left(\frac{1+\psi_{i}}{\lambda_{M} \pi_{T}}\right)^{\frac{1}{\gamma_{i}}}\left(\zeta Y_{T}\right)^{1-\frac{\gamma}{\gamma_{i}}} \mathbb{1}_{\mathcal{R}_{i, T}}, \\
& \mathcal{R}_{1, T}=\left\{\lambda_{M} \pi_{T}>b\left(\zeta Y_{T}\right)\right\}, \quad \mathcal{R}_{2, T}=\mathbb{R} \backslash \mathcal{R}_{1, T}, \\
& b\left(\zeta Y_{T}\right)=\left[\frac{\left(1+\psi_{2}\right)^{\gamma_{1}\left(\gamma_{2}-1\right)}}{\left(1+\psi_{1}\right)^{\gamma_{2}\left(\gamma_{1}-1\right)}}\left(\frac{\gamma_{1}}{\gamma_{2}}\right)^{\gamma_{1} \gamma_{2}}\right]^{\frac{1}{\gamma_{2}-\gamma_{1}}}\left(\zeta Y_{T}\right)^{-\gamma},
\end{aligned}
$$

and $\lambda_{M}>0$ solves $E_{0}\left[\pi_{T} \hat{W}_{T}\right]=w_{0}$.

Proof. Let $\zeta \equiv \bar{\zeta} W_{0} / Y_{0}$ be the normalized performance fee threshold, and let

$$
U_{T}\left(W_{T}\right) \equiv \frac{\left(f_{T} W_{T}\right)^{1-\gamma}}{1-\gamma}
$$

At $t=0$, the problem of the money manager is then:

$$
\max _{W_{T}} E_{0}\left[U_{T}\left(W_{T}\right)\right] \quad \text { s.t. } E_{0}\left[\pi_{T} W_{T}\right]=w_{0}
$$

The objective function (A10) in the manager's problem (A11) is locally non-concave in a neighborhood of $W_{T}=\zeta Y_{T}$. Standard optimization techniques cannot be applied directly to this problem. Following Basak and Makarov (2014), the first step consists in constructing the concavification $\tilde{U}_{T}(\cdot)$ of the manager's utility function $U_{T}(\cdot)$ (i.e. the smallest concave function $\tilde{U}_{T}(w)$ satisfying $\tilde{U}_{T}(w) \geq U_{T}(w)$ for all $w \geq 0$ ), restate and solve the original problem (A11) in terms of $\tilde{U}_{T}(\cdot)$.

In order to construct the concavified function, we look for functions $\underline{W}\left(\zeta Y_{T}\right), \bar{W}\left(\zeta Y_{T}\right)$, $a\left(\zeta Y_{T}\right)$ and $b\left(\zeta Y_{T}\right)$ so that (omitting the arguments for notational simplicity):

$$
\tilde{U}_{T}\left(W_{T}\right)= \begin{cases}U_{T}\left(W_{T}\right), & \text { if } W_{T}<\underline{W} \leq \zeta Y_{T}, \\ a+b W_{T}, & \text { if } \underline{W} \leq W_{T}<\bar{W}, \\ U_{T}\left(W_{T}\right), & \text { if } \zeta Y_{T} \leq \bar{W} \leq W_{T},\end{cases}
$$


and

$$
\tilde{U}_{T}^{\prime}\left(W_{T}\right)= \begin{cases}U_{T}^{\prime}\left(W_{T}\right), & \text { if } W_{T}<\underline{W} \leq \zeta Y_{T}, \\ b, & \text { if } \underline{W} \leq W_{T}<\bar{W}, \\ U_{T}^{\prime}\left(W_{T}\right), & \text { if } \zeta Y_{T} \leq \bar{W} \leq W_{T} .\end{cases}
$$

where:

$$
U_{T}^{\prime}\left(W_{T}\right)= \begin{cases}\left(1+\psi_{1}\right) W_{T}^{-\gamma_{1}}\left(\zeta Y_{T}\right)^{\gamma_{1}-\gamma}, & \text { if } W_{T}<\zeta Y_{T}, \\ \left(1+\psi_{2}\right) W_{T}^{-\gamma_{2}}\left(\zeta Y_{T}\right)^{\gamma_{2}-\gamma}, & \text { if } W_{T}>\zeta Y_{T}\end{cases}
$$

Eqs. (A12) and (A13) give us a system of 4 equations in our 4 unknowns $\underline{W}, \bar{W}, a$ and $b$ :

$$
\left\{\begin{array}{l}
a+b \underline{\underline{W}}=\frac{1}{1-\gamma} \underline{W}^{1-\gamma_{1}}\left(\zeta Y_{T}\right)^{\gamma_{1}-\gamma} \\
a+b \bar{W}=\frac{1}{1-\gamma} \bar{W}^{1-\gamma_{2}}\left(\zeta Y_{T}\right)^{\gamma_{2}-\gamma} \\
b=\left(1+\psi_{1}\right) \underline{W}^{-\gamma_{1}}\left(\zeta Y_{T}\right)^{\gamma_{1}-\gamma} \\
b=\left(1+\psi_{2}\right) \bar{W}^{-\gamma_{2}}\left(\zeta Y_{T}\right)^{\gamma_{2}-\gamma}
\end{array}\right.
$$

The solution to this system of equation yields

$$
\begin{aligned}
& b\left(\zeta Y_{T}\right)=\left[\frac{\left(1+\psi_{2}\right)^{\gamma_{1}\left(\gamma_{2}-1\right)}}{\left(1+\psi_{1}\right)^{\gamma_{2}\left(\gamma_{1}-1\right)}}\left(\frac{\gamma_{1}}{\gamma_{2}}\right)^{\gamma_{1} \gamma_{2}}\right]^{\frac{1}{\gamma_{2}-\gamma_{1}}}\left(\zeta Y_{T}\right)^{-\gamma} \\
& \underline{W}\left(\zeta Y_{T}\right)=\left[\left(\frac{1+\psi_{2}}{1+\psi_{1}}\right)^{\gamma_{2}-1}\left(\frac{\gamma_{2}}{\gamma_{1}}\right)^{\gamma_{2}}\right]^{\frac{1}{\gamma_{2}-\gamma_{1}}} \zeta Y_{T}, \\
& \bar{W}\left(\zeta Y_{T}\right)=\left[\left(\frac{1+\psi_{1}}{1+\psi_{2}}\right)^{\gamma_{1}-1}\left(\frac{\gamma_{2}}{\gamma_{1}}\right)^{\gamma_{1}}\right]^{\frac{1}{\gamma_{2}-\gamma_{1}}} \zeta Y_{T} .
\end{aligned}
$$

In order to verify that (A14) to (A16) are indeed the solutions we are after, it remains to verify that $\underline{W}$ and $\bar{W}$ satisfy the condition:

$$
\underline{W} \leq \zeta Y_{T} \leq \bar{W}
$$

which holds iff:

$$
\left[\left(\frac{\left(1+\psi_{2}\right)}{\left(1+\psi_{1}\right)}\right)^{\gamma_{2}-1}\left(\frac{\gamma_{2}}{\gamma_{1}}\right)^{\gamma_{2}}\right]^{\frac{1}{\gamma_{2}-\gamma_{1}}}<1
$$

and

$$
\left[\left(\frac{\left(1+\psi_{1}\right)}{\left(1+\psi_{2}\right)}\right)^{\gamma_{1}-1}\left(\frac{\gamma_{2}}{\gamma_{1}}\right)^{\gamma_{1}}\right]^{\frac{1}{\gamma_{2}-\gamma_{1}}}>1
$$

Since

$$
\frac{1+\psi_{2}}{1+\psi_{1}}<\left(\frac{1+\psi_{2}}{1+\psi_{1}} \frac{\gamma_{1}}{\gamma_{2}}\right)^{\gamma_{2}}
$$

and

$$
\frac{1+\psi_{1}}{1+\psi_{2}}<\left(\frac{1+\psi_{1}}{1+\psi_{2}} \frac{\gamma_{2}}{\gamma_{1}}\right)^{\gamma_{1}}
$$

both conditions indeed verify. 
We can now restate the manager's optimization problem (A11) at $t=0$ as:

$$
\max _{W_{T}} E_{0}\left[\tilde{U}_{T}\left(W_{T}\right)\right] \quad \text { s.t. } E_{0}\left[\pi_{T} W_{T}\right]=w_{0} .
$$

Attaching Lagrange multiplier $\lambda_{M}$ to the budget constraint, the solution to the concavified problem (A17) is given by the standard (state-by-state) first order condition:

$$
\tilde{U}_{T}^{\prime}\left(W_{T}\right)=\lambda_{M} \pi_{T}
$$

Using (A13):

$$
\lambda_{M} \pi_{T}= \begin{cases}\left(1+\psi_{1}\right) W_{T}^{-\gamma_{1}}\left(\zeta Y_{T}\right)^{\gamma_{1}-\gamma}, & \text { if } W_{T}<\zeta Y_{T}, \\ b, & \text { if } \underline{W} \leq W_{T}<\bar{W} \\ \left(1+\psi_{2}\right) W_{T}^{-\gamma_{2}}\left(\zeta Y_{T}\right)^{\gamma_{2}-\gamma}, & \text { if } W_{T}>\zeta Y_{T},\end{cases}
$$

which gives the manager's optimal time- $T$ AUM as:

$$
\hat{W}_{T}= \begin{cases}\left(1+\psi_{1}\right)^{\frac{1}{\gamma_{1}}}\left(\zeta Y_{T}\right)^{\frac{\gamma_{1}-\gamma}{\gamma_{1}}}\left(\lambda_{M} \pi_{T}\right)^{-\frac{1}{\gamma_{1}}}, & \text { if } W_{T}<\underline{W}, \\ W \in[\underline{W}, \bar{W}], & \text { if } \underline{W} \leq W_{T}<\bar{W}, \\ \left(1+\psi_{2}\right)^{\frac{1}{\gamma_{2}}}\left(\zeta Y_{T}\right)^{\frac{\gamma_{2}-\gamma}{\gamma_{2}}}\left(\lambda_{M} \pi_{T}\right)^{-\frac{1}{\gamma_{2}}}, & \text { if } \bar{W} \leq W_{T} .\end{cases}
$$

Using Eqs. (A14) through (A16), we note that:

$$
\hat{W}_{T}<\underline{W} \Leftrightarrow \lambda_{M} \pi_{T}>b,
$$

and

$$
\hat{W}_{T} \geq \bar{W} \Leftrightarrow \lambda_{M} \pi_{T} \leq b,
$$

which allows us to re-express (A18) as:

$$
\hat{W}_{T}=\left\{\begin{array}{lll}
\left(1+\psi_{1}\right)^{\frac{1}{\gamma_{1}}}\left(\zeta Y_{T}\right)^{\frac{\gamma_{1}-\gamma}{\gamma_{1}}}\left(\lambda_{M} \pi_{T}\right)^{-\frac{1}{\gamma_{1}}}, & \text { if } \lambda_{M} \pi_{T}>b & \left(\mathcal{R}_{1, T}\right), \\
\left(1+\psi_{2}\right)^{\frac{1}{\gamma_{2}}}\left(\zeta Y_{T}\right)^{\frac{\gamma_{2}-\gamma}{\gamma_{2}}}\left(\lambda_{M} \pi_{T}\right)^{-\frac{1}{\gamma_{2}}}, & \text { if } \lambda_{M} \pi_{T} \leq b & \left(\mathcal{R}_{2, T}\right) .
\end{array}\right.
$$

To obtain the expression for the optimal portfolio (30), we first solve for the time- $t$ optimal AUM $\hat{W}_{t}$. By no-arbitrage, the deflated wealth process $\pi_{t} \hat{W}_{t}$ is a martingale, so using (A19) the optimal wealth $\hat{W}_{t}$ for all $t \in[0, T]$ is given by:

$$
\begin{aligned}
\pi_{t} \hat{W}_{t} & =E_{t}\left[\pi_{T} \hat{W}_{T}\right] \\
\Rightarrow \hat{W}_{t} & =\hat{W}_{1, t}+\hat{W}_{2, t},
\end{aligned}
$$

where:

$$
\hat{W}_{i, t}=\pi_{t} E_{t}\left[\left(1+\psi_{i}\right)^{\frac{1}{\gamma_{i}}}\left(\zeta Y_{T}\right)^{\frac{\gamma_{i}-\gamma}{\gamma_{i}}}\left(\lambda_{M} \pi_{T}\right)^{-\frac{1}{\gamma_{i}}} \mathbb{1}_{\mathcal{R}_{i}}\right]
$$


To compute the expectation on the RHS, we need a characterization of the state-price deflator (SPD) $\pi_{T}$ and the benchmark value $Y_{T}$. To this aim, we first define the vector process $\tilde{B}=$ $\left(\tilde{B}_{0}, \tilde{B}_{1}, \ldots, \tilde{B}_{n},\right)^{\prime}$ by $\tilde{B}_{0}=\mathbf{0}$ (where $\mathbf{0}$ is the $(n+1)$-dimensional column vector of zeros) and:

$$
d \tilde{B}_{t}=\kappa_{t} d t+d B_{t}, \quad t \in[0, T]
$$

where $\kappa$ is the "multivariate appraisal ratio" defined in Section 3. Stacking the dynamics of prices in a column vector $d S=\left(d S_{0}, d S_{1}, \ldots, d S_{n}\right)^{\prime}$, we can write $d S$ as:

$$
\begin{aligned}
d S_{t} & =I\left[S_{t}\right]\left(\left(\bar{\mu}_{t}+\alpha_{t}\right) d t+\Sigma_{t} d B_{t}\right) \\
& =I\left[S_{t}\right]\left(\bar{\mu}_{t} d t+\Sigma_{t} d \tilde{B}_{t}\right) .
\end{aligned}
$$

Let $\mathbb{P}$ denote the actual (objective) probability measure under which $B$ is a standard Brownian motion vector process. We show below that $\tilde{B}$ is a standard Brownian motion vector process under an equivalent probability measure $\tilde{\mathbb{P}}$. Eq. (A23) then indicates that the risky assets show no mispricing under the equivalent probability $\tilde{\mathbb{P}}$, because their mean returns equal the "fundamental" returns $\bar{\mu}$ and $\alpha_{i}$ is identically equal to 0 for all $i$. In this "no-mispricing" economy, markets are still complete $(n+1$ risky asset prices $S$ driven by $n+1$ independent Brownian motions $\tilde{B})$, the vector of market prices of risk is $\tilde{\eta} \equiv \Sigma^{-1}(\bar{\mu}-r)$, and investors see financial markets as driven by a unique SPD

$$
\tilde{\pi}_{t}=\exp \left\{-r t-\frac{1}{2} \int_{0}^{t}\left\|\tilde{\eta}_{s}\right\|^{2} d s-\int_{0}^{t} \tilde{\eta}_{s}^{\prime} d \tilde{B}_{s}\right\}
$$

The prove that $\tilde{B}$ is a $\tilde{\mathbb{P}}$-standard Brownian motion process, define the process $\xi_{t}$ on $[0, T]$ by:

$$
\xi_{t} \equiv \exp \left\{-\frac{1}{2} \int_{0}^{t}\left\|\kappa_{s}\right\|^{2} d s-\int_{0}^{t} \kappa_{s}^{\prime} d B_{s}\right\}
$$

so that $\xi_{0}=1$. It can be verified that $\xi$ is a $\mathbb{P}$-martingale. ${ }^{41}$ Define the measure $\tilde{\mathbb{P}}$ on the filtration $\mathcal{F}_{T}$ generated by the prices processes $\left(S_{t}: 0 \leq t \leq T\right)$ by $\xi_{T}=d \tilde{\mathbb{P}} / d \mathbb{P}$, on $\mathcal{F}_{T}$. By an application of the Girsanov's Theorem, $\tilde{\mathbb{P}}$ is a probability measure equivalent to $\mathbb{P}$, and $\tilde{B}$ is a $(n+1)$-dimensional standard Brownian motion process under $\tilde{\mathbb{P}}$.

For the securities market (16)-(21), Sotes-Paladino (2017) then characterizes $\pi_{t}$ from $\tilde{\pi}_{t}$ and $\xi_{t}$ as:

$$
\pi_{t}=\tilde{\pi}_{t} \xi_{t}
$$

where for $0 \leq t \leq T$

$$
\tilde{\pi}_{t}=\tilde{\pi}_{s} \exp \left\{-r(t-s)-\frac{\gamma^{2}}{2} \delta_{0} V_{T}^{-1}\left(V_{s}-V_{t}\right) V_{T}^{-1} \delta_{0}^{\prime}+\gamma \delta_{0} V_{T}^{-1}\left(\kappa_{t}-\kappa_{s}\right)\right\},
$$

\footnotetext{
${ }^{41}$ Specifically, the Novikov condition $E\left[\exp \left\{\frac{1}{2} \int_{0}^{T}\left\|\kappa_{s}\right\|^{2} d s\right\}\right]<\infty$ can be verified to be satisfied.
} 


$$
\xi_{t}=\frac{\xi_{s}}{\sqrt{\prod_{0}^{n}\left(1+d_{j, s}(t-s)\right)}} \exp \left\{-\frac{1}{2}\left(\kappa_{t}^{\prime} V_{t}^{-1} \tilde{\theta}_{t}-\kappa_{s}^{\prime} V_{s}^{-1} \tilde{\theta}_{s}\right)-\frac{1}{2} \theta^{\prime}\left(B_{t}-B_{s}\right)\right\}
$$

where $\tilde{\pi}_{0}=\xi_{0}=1$, and $\tilde{\theta}_{t} \equiv \theta-\kappa_{t}$.

We can write the dynamics (25) of $Y$ under the equivalent "no mispricing" probability $\tilde{\mathbb{P}}$ as:

$$
d Y_{t}=Y_{t}\left(r+\left(\phi^{Y}\right)^{\prime} \Sigma_{t} \tilde{\eta}_{t}\right) d t+Y_{t}\left(\phi^{Y}\right)^{\prime} \Sigma_{t} d \tilde{B}_{t}
$$

where $\tilde{B}=\kappa_{t} d t+d B_{t}$ is a standard Brownian motion vector process under $\tilde{\mathbb{P}}$. Eq. (A29) implies:

$$
\begin{aligned}
Y_{T} & =Y_{t} \exp \left\{r \tau+\gamma\left(\phi^{Y}\right)^{\prime}\left(\int_{t}^{T} \Sigma_{s}^{2} d s\right) e_{M}-\frac{1}{2}\left(\phi^{Y}\right)^{\prime}\left(\int_{t}^{T} \Sigma_{s}^{2} d s\right) \phi^{Y}+\left(\phi^{Y}\right)^{\prime} \int_{t}^{T} \Sigma_{s} d \tilde{B}_{s}\right\} \\
& =Y_{t} \exp \left\{r \tau+\left(\phi^{Y}\right)^{\prime} \Delta V_{T}^{-1}\left(V_{t}-V_{T}\right) V_{T}^{-1} \Delta^{\prime}\left(\gamma-\frac{1}{2} \phi^{Y}\right)\left(\phi^{Y}\right)^{\prime} \Delta V_{T}^{-1}\left(\tilde{\theta}_{T}-\tilde{\theta}_{t}\right)\right\},
\end{aligned}
$$

where we used $d V_{s}=-V_{s}^{2} d s$ and $d \tilde{\theta}_{s}=V_{s} d \tilde{B}_{s}$ to solve for the integrals in (A30). Defining:

$$
\zeta_{0} \equiv\left[\frac{\left(1+\psi_{2}\right)^{\gamma_{1}\left(\gamma_{2}-1\right)}}{\left(1+\psi_{1}\right)^{\gamma_{2}\left(\gamma_{1}-1\right)}}\left(\frac{\gamma_{1}}{\gamma_{2}}\right)^{\gamma_{1} \gamma_{2}}\right]^{\frac{1}{\gamma_{2}-\gamma_{1}}}
$$

we can express $b\left(\zeta Y_{T}\right)=\zeta_{0}\left(\zeta Y_{T}\right)^{-\gamma}$. Region $\mathcal{R}_{1, T}$ is then given by:

$$
\lambda_{M} \pi_{T}>b\left(\zeta Y_{T}\right) \Leftrightarrow \lambda_{M} \pi_{T}>\zeta_{0}\left(\zeta Y_{T}\right)^{-\gamma}
$$

Replacing (A26) and (A30) in this condition we obtain, after much simplification,

$$
\left\|I_{d_{T}}^{-\frac{1}{2}}\left(\bar{\theta}_{T}-\bar{\theta}-\gamma P\left(\delta_{M}^{\prime}-\Delta \phi^{Y}\right)\right)\right\|>\Gamma
$$

where

$$
\begin{aligned}
\Gamma^{2}= & \left\|I_{d_{0}}^{-\frac{1}{2}}\left(\bar{\theta}_{0}-\bar{\theta}-\gamma P\left(\delta_{M}^{\prime}-\Delta \phi^{Y}\right)\right)\right\|-\left\|I_{d_{0}}^{-\frac{1}{2}}\left(\bar{\theta}+\gamma P\left(\delta_{M}^{\prime}-\Delta \phi^{Y}\right)\right)\right\|^{2} \\
& +\left\|I_{d_{T}}^{-\frac{1}{2}}\left(\bar{\theta}+\gamma P\left(\delta_{M}^{\prime}-\Delta \phi^{Y}\right)\right)\right\|^{2}-2 \gamma\left(\delta_{M}-\left(\phi^{Y}\right)^{\prime} \Delta\right) P^{\prime} \bar{\theta}_{0} T-\|\bar{\theta}\| T \\
& -2(\gamma-1) r T-2 \gamma\left(\phi^{Y}\right)^{\prime} \Delta V_{T}^{-1}\left(V_{0}-V_{T}\right) V_{T}^{-1}\left(\gamma \delta_{M}^{\prime}-\frac{1}{2} \Delta^{\prime} \phi^{Y}\right) \\
& +\gamma^{2} \delta_{M} V_{T}^{-1}\left(V_{0}-V_{T}\right) V_{T}^{-1} \delta_{M}^{\prime}+2 \ln \left[\frac{\zeta_{0} \prod_{0}^{n} \sqrt{1+d_{j, 0} T}}{\lambda_{M}\left(\zeta Y_{0}\right)^{\gamma}}\right] \\
= & \left(\bar{\eta}_{0}^{\prime}-\gamma\left(\phi^{Y}\right)^{\prime} \bar{\Sigma}_{0}\right) I_{d_{0}}^{-1}\left(\bar{\eta}_{0}-\gamma \bar{\Sigma}_{0}^{\prime} \phi^{Y}\right)-2(\gamma-1)\left(r+\frac{\gamma}{2}\left(\phi^{Y}\right)^{\prime} \bar{\Sigma}_{0}\left(I+T I_{d_{0}}\right)^{-1} \bar{\Sigma}_{0}^{\prime} \phi^{Y}\right) T \\
& +2 \ln \left[\frac{\zeta_{0} \prod_{0}^{n} \sqrt{1+d_{j, 0} T}}{\lambda_{M}\left(\zeta Y_{0}\right)^{\gamma}}\right]
\end{aligned}
$$

and $\bar{X}^{\prime}$ and $\bar{x}$ denote, respectively, the rotated matrix $\bar{X}^{\prime} \equiv P X^{\prime}$ and vector $\bar{x} \equiv P x$ as introduced in Section 3.2.

We can now compute the expectation on the RHS of (A21). Indeed, (A26) to (A28) and 
(A30) indicate that conditional on time $t, \pi_{T}$ and $Y_{T}$ follow multivariate log-normal distributions. Computing the expectation by direct integration against the multivariate normal density on the region defined by (A33) gives, after lengthy algebraic manipulation,

$$
\begin{aligned}
\hat{W}_{i, t}= & \left(\frac{1+\psi_{i}}{\lambda_{M} \pi_{t}}\right)^{\frac{1}{\gamma_{i}}}\left(\zeta Y_{t}\right)^{\frac{\gamma_{i}-\gamma}{\gamma_{i}}} \prod_{j=0}^{n} \sqrt{\frac{\left(1+d_{j, t} \tau\right)^{\frac{1}{\gamma_{i}}}}{1+\frac{d_{j, t}}{\gamma_{i}}}} \\
& \times \exp \left\{-\frac{\gamma-1}{\gamma_{i}}\left[r+\frac{1}{2} \frac{\gamma_{i}-\gamma}{\gamma_{i}-1}\left(\phi^{Y}\right)^{\prime} \bar{\Sigma}_{t}\left(I+\tau I\left[d_{t}\right]\right)^{-1} \bar{\Sigma}_{t}^{\prime} \phi^{Y}\right] \tau\right. \\
& \left.-\frac{1}{2 \gamma_{i}}\left(1-\frac{1}{\gamma_{i}}\right)\left(\bar{\eta}_{t}^{\prime}-\frac{\gamma_{i}-\gamma}{\gamma_{i}-1}\left(\phi^{Y}\right)^{\prime} \bar{\Sigma}_{t}\right)\left(I+\frac{\tau}{\gamma_{i}} I\left[d_{t}\right]\right)^{-1}\left(\bar{\eta}_{t}-\frac{\gamma_{i}-\gamma}{\gamma_{i}-1} \bar{\Sigma}_{t}^{\prime} \phi^{Y}\right) \tau\right\} \Pi_{i, t},
\end{aligned}
$$

where

$$
\begin{aligned}
& \Pi_{i, t} \equiv \operatorname{Prob}\left(\overline{\mathcal{R}}_{i, t}\right)=\int_{\overline{\mathcal{R}}_{i, t}} \prod_{j=0}^{n} n\left(z_{j}\right) d z_{0} d z_{1} \ldots d z_{n} \\
& \overline{\mathcal{R}}_{i, t} \equiv\left\{\begin{array}{l}
\left\|\left(I+\frac{\tau}{\gamma_{1}} I\left[d_{t}\right]\right)^{-\frac{1}{2}}\left(\tau I\left[d_{t}\right]\right)^{\frac{1}{2}}\left(z-A_{1, t}\right)\right\|>\Gamma, \quad i=1 \\
\left\|\left(I+\frac{\tau}{\gamma_{2}} I\left[d_{t}\right]\right)^{-\frac{1}{2}}\left(\tau I\left[d_{t}\right]\right)^{\frac{1}{2}}\left(z-A_{2, t}\right)\right\| \leq \Gamma, \quad i=2
\end{array}\right. \\
& A_{i, t} \equiv \gamma\left(I+\frac{\tau}{\gamma_{i}} I\left[d_{t}\right]\right)^{-\frac{1}{2}}\left(I+\tau I\left[d_{t}\right]\right)^{\frac{1}{2}}\left(\tau^{\frac{1}{2}} I\left[d_{t}\right]\right)^{-1}\left(I+\frac{\tau}{\gamma} I\left[d_{t}\right]\right) \bar{\Delta}^{\prime}\left(\phi_{t}^{N}-\phi^{Y}\right),
\end{aligned}
$$

$n(\cdot)$ is the standard normal density function, and $z \equiv\left(z_{0}, z_{1}, \ldots, z_{n}\right)^{\prime}$.

In order to derive the investment policy (30) replicating the optimal portfolio value (A20), note that this can be rewritten as $\hat{W}_{t}=h\left(t, Y_{t}, \pi_{t}, \bar{\eta}_{t}, \Pi_{i, t}\right)$, some function $h \in C^{1,2}$. Applying Itô's Lemma the diffusion term $\sigma_{\hat{W}}$ of $d \hat{W}_{t}$ is:

$$
\sigma_{\hat{W}}=h_{Y} \sigma_{Y}+h_{\pi} \sigma_{\pi}+h_{\bar{\eta}} \sigma_{\bar{\eta}}+h_{\Pi_{i}} \sigma_{\Pi_{i}},
$$

where $h_{x}$ denotes the partial derivative of $h$ w.r.t. $x$ and $\sigma_{X}$ is the diffusion term in the SDE characterizing the dynamics of the process $X$. Computing the diffusion terms in (A38) explicitly and equating the result to the diffusion term of $W_{t}$ in (24) gives the optimal portfolio (30)-(31).

To obtain $\hat{W}_{t}$ in Proposition 1, we specialize (A35)-(A37) to $n=0$ to get:

$$
\begin{aligned}
\hat{W}_{i, t}= & \left(\frac{1+\psi_{i}}{\lambda_{M} \pi_{t}}\right)^{\frac{1}{\gamma_{i}}}\left(\zeta Y_{t}\right)^{1-\frac{\gamma}{\gamma_{i}}} \sqrt{\frac{\left(1+v_{t} \tau\right)^{\frac{1}{\gamma_{i}}}}{1+\frac{v_{t}}{\gamma_{i}} \tau}} \times \exp \left\{-\frac{\gamma-1}{\gamma_{i}}\left[r+\frac{1}{2} \frac{\gamma_{i}-\gamma}{\gamma_{i}-1} \frac{\left(\sigma_{t} \phi^{Y}\right)^{2}}{1+v_{t} \tau}\right] \tau\right. \\
& \left.-\frac{1}{2 \gamma_{i}}\left(1-\frac{1}{\gamma_{i}}\right) \frac{\left(\eta_{t}-\frac{\gamma_{i}-\gamma}{\gamma_{i}-1} \sigma_{t} \phi^{Y}\right)^{2}}{1+\frac{v_{t}}{\gamma_{i}} \tau} \tau\right\} \Pi_{i, t}
\end{aligned}
$$

where $\Pi_{1, t} \equiv N\left(\underline{d}_{1, t}\right)+1-N\left(\bar{d}_{1, t}\right), \Pi_{2, t} \equiv N\left(\bar{d}_{2, t}\right)-N\left(\underline{d}_{2, t}\right), N(\cdot)$ is the standard normal 
cumulative distribution function, and

$$
\underline{d}_{i, t} \equiv \gamma \sqrt{\frac{1+v_{t} \tau}{1+\frac{v_{t}}{\gamma_{i}} \tau}} \frac{1+\frac{v_{t}}{\gamma} \tau}{v_{t} \sqrt{\tau}} \delta\left(\phi_{\gamma, t}^{N}-\phi^{Y}\right)-\sqrt{\frac{1+\frac{v_{t}}{\gamma_{i}} \tau}{v_{t} \tau}} \Gamma, \quad \bar{d}_{i, t} \equiv \underline{d}_{i, t}+2 \sqrt{\frac{1+\frac{v_{t}}{\gamma_{i}} \tau}{v_{t} \tau}} \Gamma .
$$

Applying Itô's Lemma to compute the diffusion term $\sigma_{\hat{W}}$ of $d \hat{W}_{t}$, and equating the result to the diffusion of $W_{t}$ in (7) (equivalently, specializing (30)-(31) to the case $n=0$ and rearranging) gives the optimal portfolio (13)-(14) in Proposition 1. 


\section{B Parameterization of Money Managers' Incentives}

\section{B.1 Incentive Fee Schedules of Hedge Funds}

The typical fee contract in the hedge fund industry ("two-twenty") stipulates a $2 \%$ management fee plus an incentive fee equal to $20 \%$ of profits beyond a designated benchmark performance. The benchmark is usually a money market rate such as LIBOR plus a spread, consistent with the typical goal in the industry of delivering "absolute returns" in all market conditions. ${ }^{42}$

To capture these incentives, we set the benchmark weight in the risky assets to zero $\left(\phi^{Y}=0\right)$, in which case $Y_{T}=Y_{0} e^{r T}$. We define the continuously-compounded rates $r_{T} \equiv \ln \left(R_{T}\right) / T, r_{T}^{Y} \equiv$ $\ln \left(R_{T}^{Y}\right) / T=r$, and set the threshold performance $\bar{\zeta}$ to equal $e^{h T}$, for the spread (hurdle rate) $h \geq 0$. For $\psi_{1}=0$, and for any $\alpha>0$, we can then write:

$$
k\left(\frac{R_{T}}{\bar{\zeta} R_{T}^{Y}}\right)^{\alpha}=k e^{\alpha\left(r_{T}-(r+h)\right) T} .
$$

A first-order approximation of the RHS of (B1) around $r_{T}=r+h$ gives:

$$
k\left(\frac{R_{T}}{\bar{\zeta} R_{T}^{Y}}\right)^{\alpha} \approx k+k \alpha\left(r_{T}-(r+h)\right) T
$$

We apply (B2) to the two terms in the RHS of (9) and set $\psi_{1}=0$, which implies a fee rate:

$$
f_{T} \approx k+k \psi_{2}\left(r_{T}-(r+h)\right)^{+} T
$$

where $x^{+} \equiv \max (0, x)$. From equation (B3), we can calibrate a two-twenty contract with no hurdle rate within our setup by setting, e.g., $k=1, k T \psi_{2}=5$, and $(r+h)=1.5 \%+0 \%=1.5 \%{ }^{43}$

\section{B.2 Flow-Performance Relationship of Mutual Funds}

In the mutual fund industry, convex incentives can arise implicitly from the relation between a fund's performance relative to its benchmark and its investors' share purchases and redemptions. In the U.S., mutual funds typically charge a management fee proportional to AUM but no incentive fees. Let $m$ be the management fee. An extensive literature (see, e.g., Chevalier and Ellison (1997), and Sirri and Tufano (1998)) documents that mutual fund inflows after positive benchmark-adjusted performance largely exceed outflows following negative benchmark-

\footnotetext{
42 The fee structure often includes a "high-water mark" condition so that the fund has to recover losses before any incentive fee can be charged following a year in which the fund declines in value. Such provisions may reduce the long-term risk-seeking incentives of a hedge fund manager, as analyzed by Hodder and Jackwerth (2007), Panageas and Westerfield (2009), and Drechsler (2013). Since we focus on the trading behavior of the manager over short-horizons, we follow Buraschi, Kosowski, and Sritrakul (2011) and assume that the high-water mark is prespecified at the beginning of the period, and allow differences in high-water marks by varying the spread parameter $\bar{\zeta}$ in (9).

${ }^{43}$ Given the exponential form of the performance fee (9) in the outperformance region, $\psi_{2}=5$ can reflect a performance fee that is a large multiple (e.g., 10) of the base management fee, as observed in practice, for a wide range of relative performance values.
} 
adjusted returns. In the model, we capture the ensuing convexity in the so-called flow-to-relative performance relationship (FPR) as follows. At $t=T$, mutual fund investors purchase or redeem additional fund shares depending on the manager's performance relative to the benchmark $Y$ at the flow rate $q_{T}$ :

$$
q_{T}=\underline{q}\left(\frac{R_{T}}{\bar{\zeta} R_{T}^{Y}}\right)^{\psi_{1}} \mathbb{1}_{\left\{R_{T}<\bar{\zeta} R_{T}^{Y}\right\}}+\underline{q}\left(\frac{R_{T}}{\bar{\zeta} R_{T}^{Y}}\right)^{\psi_{2}} \mathbb{1}_{\left\{R_{T} \geq \bar{\zeta} R_{T}^{Y}\right\}},
$$

with $\underline{q}>0$. Setting $k \equiv m \underline{q}$, it is easy to see that the mutual fund manager's fee rate $f_{T}=m q_{T}$ fits specification (9). This functional form allows flows to be sensitive (and potentially locally concave for $\psi_{1}<1$ ) to medium and low relative performance. For $\psi_{1}<\psi_{2}, f_{T}$ reflects the asymmetric sensitivity of flows to performance according to which outperforming funds receive a disproportionally high amount of inflows. ${ }^{44}$ The FPR (B4) can also capture linear relationships $\left(\psi_{1}=\psi_{2}=1\right)$, log-linear relationships $\left(\psi_{1}=\psi_{2} \neq 1\right)$, as well as no relationship $\left(\psi_{1}=\psi_{2}=0\right)$.

\footnotetext{
${ }^{44}$ While many empirical studies document no sensitivity of flows to poor relative past performance (e.g. Sirri and Tufano (1998)), many others (e.g. Huang, Wei, and Yan (2007)) find it is positive, although lower than the sensitivity to medium or high relative returns.
} 


\section{Benchmarking vs. Incentive Convexity and Robustness}

In Sections 2 and 3 we compare the impact of convexity versus benchmarking effects on the manager's trading of a mispriced asset through its $A P H, R P H$ and $R S D$ demands. We next perform a similar analysis by comparing the investment behavior of managers with vs. without kinked incentives, when they face the same performance concern relative to the mispriced asset. We argue that the results of this analysis have empirical relevance, as they highlight the difficulties, without accounting for incentive convexity, to rationalize the long-short convergence trade strategies that sophisticated investors use in practice.

To remove the kink in the incentives of a manager with relative performance concerns, we set $0<\psi_{1}=\psi_{2}$ in (9). We show the results in Figure C.1, where we plot the trading in assets 0 (the benchmark) and 1 (the non-benchmark asset) of the "benchmarked" investors with (a MF S manager) and without kinked incentives. For ease of comparison, we adopt the correlated mispricing scenario of Section 3.3.2. To illustrate the robustness of our results to different risk aversion values, we further assume a more risk-tolerant manager $(\gamma=3)$ than in Sections 2 and 3.

In line with our previous analysis, we see that convex incentives can lessen the manager's over-investment in overvalued benchmark assets $(i=0)$ but exacerbate it in overvalued nonbenchmark assets $(i=1)$. Indeed, the top row of Fig. C.1 shows that the MF S manager typically overweights the overvalued benchmark asset 0 in her portfolio less intensively than a comparable manager with the same benchmark but no kinked incentives. In contrast, the bottom row indicates that incentive convexity can lead a manager to overweight a negative-risk premium asset more when it does not belong to the benchmark. The difference is particularly striking in situations where both benchmark and non-benchmark assets are overvalued, but the latter relatively less so. In these situations, the benchmarked managers with and without convex incentives can adopt opposite positions in the non-benchmark asset. Whereas the MF S manager holds the non-benchmark asset long, the benchmarked manager without convex incentives holds a long position in the relatively more expensive benchmark asset and sells the cheaper, nonbenchmark asset short. 

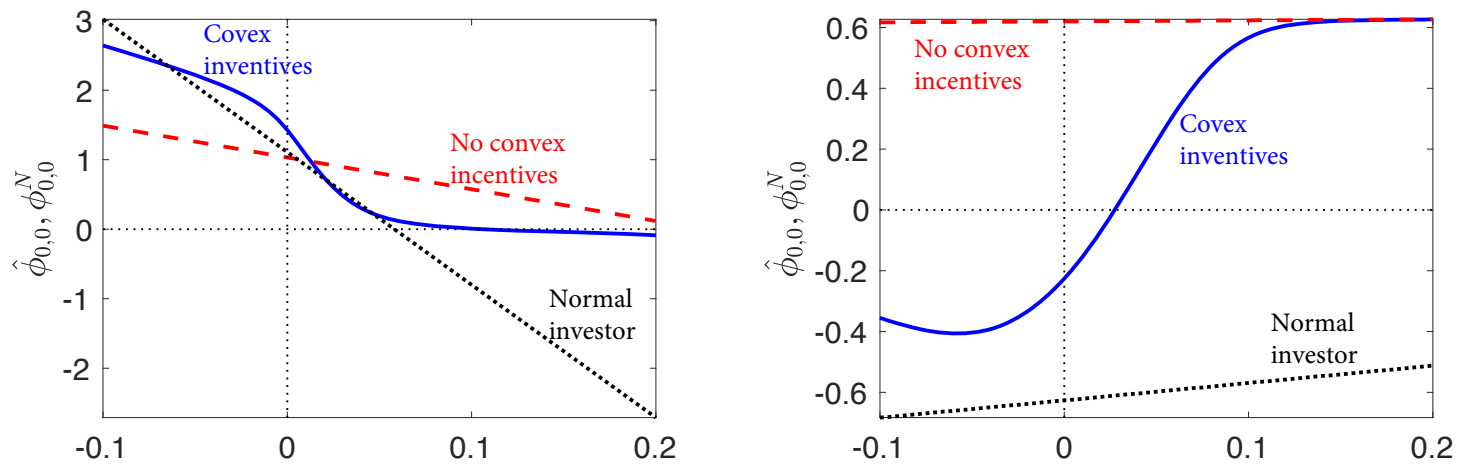

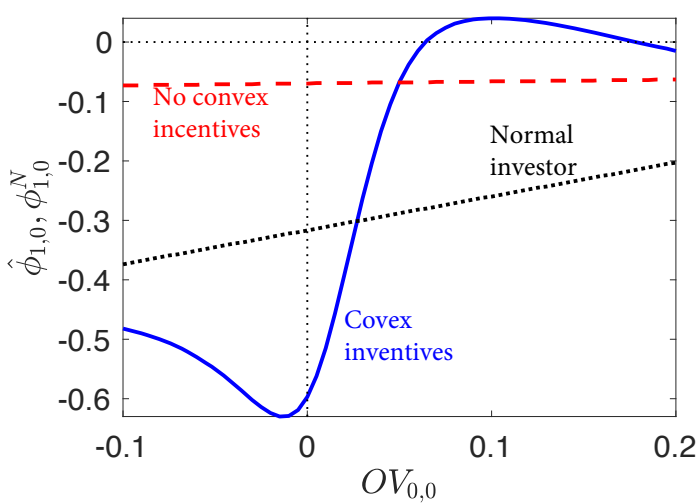

(a) Mispricing of Asset $i=0\left(O V_{1,0}=0.03\right)$

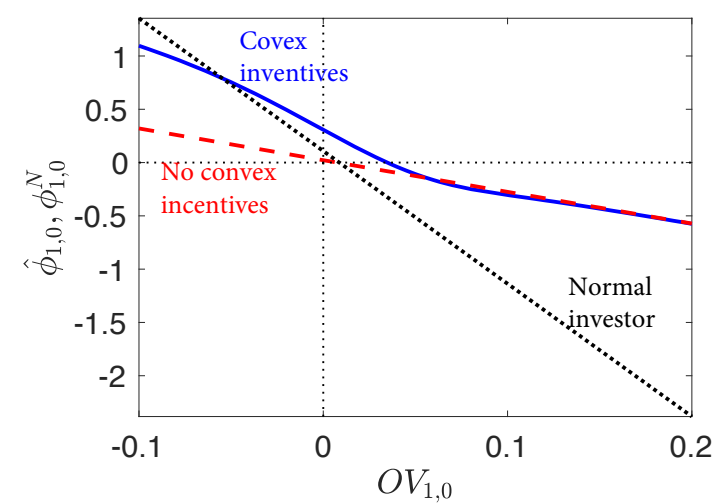

(b) Mispricing of Asset $i=1\left(O V_{0,0}=0.09\right)$

\section{Figure C.1: Convexity versus Benchmarking Effects}

This figure compares the investment policy of a MF S manager with versus without kinked incentives, at different initial mispricing states $O V_{0,0}$ (Panel 1(a)) and $O V_{1,0}$ (Panel 1(b)). Across panels, we illustrate the initial portfolio weights allocated to asset 0 (top row) and 1 (bottom row) of the MF S manager with kinked incentives $\left(\psi_{1}=0, \psi_{2}=5, \bar{\zeta}=1\right.$, solid blue line), the MF S manager without kinked incentives $\left(\psi_{1}=5, \psi_{2}=5\right.$, dashed red line) and the normal investor (dotted black line). The relative risk aversion parameter is: $\gamma=3$. The rest of the parameter values are as in Figure 3 . 